TRANSACTIONS OF THE

AMERICAN MATHEMATICAL SOCIETY

Volume 361, Number 2, February 2009, Pages 979-1005

S 0002-9947(08)04549-2

Article electronically published on August 18, 2008

\title{
THE PLURICOMPLEX POISSON KERNEL FOR STRONGLY CONVEX DOMAINS
}

\author{
FILIPPO BRACCI, GIORGIO PATRIZIO, AND STEFANO TRAPANI
}

\begin{abstract}
Let $D$ be a bounded strongly convex domain in the complex space of dimension $n$. For a fixed point $p \in \partial D$, we consider the solution of a homogeneous complex Monge-Ampère equation with a simple pole at $p$. We prove that such a solution enjoys many properties of the classical Poisson kernel in the unit disc and thus deserves to be called the pluricomplex Poisson kernel of $D$ with pole at $p$. In particular we discuss extremality properties (such as a generalization of the classical Phragmen-Lindelof theorem), relations with the pluricomplex Green function of $D$, uniqueness in terms of the associated foliation and boundary behaviors. Finally, using such a kernel we obtain explicit reproducing formulas for plurisubharmonic functions.
\end{abstract}

\section{INTRODUCTION}

During the past decades the study of pluripotential theory and its applications played a central role in complex analysis in several variables. In particular, since the basic work of Siciak [31] and Bedford and Taylor [7, 8], great effort was made to understand the complex Monge-Ampère operator and the associated generalized Dirichlet problems (for instance, see [15, 20] and the references therein).

Let $D \subset \mathbb{C}^{n}$ be a bounded convex domain with $z_{0} \in D$. From the work of Lempert [21, 24] and Demailly [15] it turned out that the homogeneous MongeAmpère equation

$$
\left\{\begin{array}{l}
u \in \operatorname{Psh}(D), \\
(\partial \bar{\partial} u)^{n}=0 \text { in } D \backslash\left\{z_{0}\right\}, \\
\lim _{z \rightarrow x} u(z)=0 \text { for all } x \in \partial D, \\
u(z)-\log \left|z-z_{0}\right|=O(1) \quad \text { as } z \rightarrow z_{0}
\end{array}\right.
$$

has a solution $L_{D, z_{0}}$ which is continuous in $D \backslash\left\{z_{0}\right\}$ (actually it is smooth there if $D$ is strongly convex with a smooth boundary) and unique.

The function $L_{D, z_{0}}$ shares many properties with the Green function for the unit disc $\mathbb{D} \subset \mathbb{C}$. For instance, from an analytic point of view it can be used to reproduce continuous plurisubharmonic functions (see [15] or Section 8), while from a geometrical point of view, its level sets are boundaries of Kobayashi balls centered at $z_{0}$ and its associated foliation is the singular pencil of complex geodesics passing through $z_{0}$. Thus it can be successfully used in questions such as classification of domains or biholomorphisms (see, e.g., 28], 29], 9]). Therefore, such a function deserves the name of pluricomplex Green's function.

Received by the editors September 26, 2006 and, in revised form, May 2, 2007.

2000 Mathematics Subject Classification. Primary 32W20, 32U35. 
In [11] the first and second named authors concentrated on studying a homogeneous Monge-Ampère equation with a simple singularity at the boundary. Namely, the following result has been proved:

Theorem 0.1. Let $D \subset \mathbb{C}^{n}$ be a bounded strongly convex domain with smooth boundary and let $p \in \partial D$. The Monge-Ampère equation

$$
\left\{\begin{array}{l}
u \in \operatorname{Psh}(D), \\
(\partial \bar{\partial} u)^{n}=0 \quad \text { in } D, \\
u<0 \text { in } D, \\
u(z)=0 \quad \text { for } z \in \partial D \backslash\{p\}, \\
u(z) \approx\|z-p\|^{-1} \quad \text { as } z \rightarrow p \text { non-tangentially }
\end{array}\right.
$$

has a solution $\Omega_{D, p} \in C^{\infty}(\bar{D} \backslash\{p\})$ such that $d\left(\Omega_{D, p}\right)_{z} \neq 0$ and $\left(\partial \bar{\partial} \Omega_{D, p}\right)^{n-1}(z) \neq 0$ for all $z \in \bar{D} \backslash\{p\}$. Moreover the level sets of $\Omega_{D, p}$ are boundaries of horospheres of $D$ with center $p$.

Here $\operatorname{Psh}(D)$ denotes the real cone of plurisubharmonic functions in $D$, and horospheres are the "limits of Kobayashi balls" introduced by Abate [1, 2] and coincide with the sub-level sets of Busemann functions of geodesics whose closure contains $p$ (see [12]). The function $\Omega_{D, p}$ has been defined by means of the boundary spherical representation of Chang-Hu-Lee [13] (see Section 11). In 11], among other things, it has been proved that $\Omega_{D, p}$ can be used to characterize biholomorphisms and that its associated foliation is the fibration of complex geodesics of $D$ whose closure contains $p$.

The aim of this paper is to study the properties of $\Omega_{D, p}$ in depth. We will show that $\Omega_{D, p}$ shares many properties with the Poisson kernel for the unit disc $\mathbb{D}$, and therefore it deserves the name of pluricomplex Poisson kernel of $D$ with singularity at $p \in \partial D$.

In more detail, we show that a version of the classical Phragmen-Lindelöf theorem on the growth of subharmonic functions in $\mathbb{D}$ holds for plurisubharmonic functions in $D$, proving that $\Omega_{D, p}$ is the maximal element of the family

$$
\left\{\begin{array}{l}
u \in \operatorname{Psh}(D), \\
\limsup _{z \rightarrow x} u(z) \leq 0 \quad \text { for all } x \in \partial D \backslash\{p\}, \\
\liminf _{t \rightarrow 1}|u(\gamma(t))(1-t)| \geq 2 \operatorname{Re}\left(\left\langle\gamma^{\prime}(1), \nu_{p}\right\rangle^{-1}\right),
\end{array}\right.
$$

where $\nu_{p}$ is the unit outward normal to $\partial D$ at $p$ and $\gamma$ is any $C^{1}$-curve in $D$ such that $\gamma(1)=p$ and $\gamma^{\prime}(1) \notin T_{p} \partial D$ (see Section 5 ). In due course we will find the exact behavior of $\Omega_{D, p}(z)$ as $z$ goes to $p$ along non-tangential directions to $\partial D$ at $p$ (see Corollary [5.3).

Next, we deal with uniqueness properties of $\Omega_{D, p}$. These are essentially of two types: analytic and geometric. From an analytic point of view we show that $\Omega_{D, p}$ is the only solution of the homogeneous Monge-Ampère equation which is zero on $\partial D \backslash\{p\}$ and behaves like $\Omega_{D, p}$ as $z$ tends to $p$ (see Theorem 7.1). This is analogous to the uniqueness statement for the pluricomplex Green function, except that the behavior of $\Omega_{D, p}$ near $p$ is universal only along non-tangential directions, but it might depend on the domain $D$ itself along other directions. From a geometrical point of view we show that $\Omega_{D, p}$ is the only $C^{2}$ solution (up to multiplication by constants) of the homogeneous Monge-Ampère equation which is zero on $\partial D \backslash\{p\}$ 
and whose associated foliation is the fibration of $D$ in complex geodesics whose closure contains $p$ (see Theorem 7.3). This fact is then used to show other interesting characterizations of $\Omega_{D, p}$ both in terms of its level sets (see Proposition 7.4) and in terms of its behavior under pull-back with holomorphic self-maps of $D$ (see Proposition 7.5).

We also show in Theorem 6.1 that $L_{D, z_{0}}$ and $\Omega_{D, p}$ have the same relationship as the Green function and the Poisson kernel in $\mathbb{D}$, namely

$$
\Omega_{D, p}\left(z_{0}\right)=-\frac{\partial L_{D, z_{0}}}{\partial \nu_{p}}(p) .
$$

This is used to write explicitly the "noyaux de Poisson pluricomplexes canonique" of Demailly [15] and, applying his theory, to obtain a somewhat explicit reproducing formula for continuous plurisubharmonic functions of $D$ in terms of $L_{D, z_{0}}$ and $\Omega_{D, p}$ (see Theorem 8.2). In particular, for pluriharmonic functions $F \in C^{0}(\bar{D})$ we obtain the following formula which is analogous to that for harmonic functions in the disc:

$$
F(z)=\int_{p \in \partial D}\left|\Omega_{D, p}(z)\right|^{n} F(p) \omega_{\partial D}(p),
$$

where $\omega_{\partial D}$ is a positive real $(2 n-1)$-form on $\partial D$ which depends only on $D$.

As a spin off result, using the properties of $\Omega_{D, p}$, we also prove that horospheres are (smooth and) strongly convex away from their center (see Theorem 4.1).

The proofs of the previous properties of $\Omega_{D, p}$ are based on a mix of different techniques. In particular we will make a strong use of families of complex geodesics and their regularity properties. Thus in Section 2 we deal with regularity for such families, gathering some known but disperse information and proving the precise results needed for our arguments. In particular, using a suitable "attached analytic discs" approach, we prove (Theorem 2.1) that the set of complex geodesics in $D$ is a finite dimensional closed submanifold in the open set of the complex Banach space $\mathcal{O}^{k+\alpha}\left(\overline{\mathbb{D}}, \mathbb{C}^{n}\right)$ made of non-constant holomorphic attached discs whose first $k$-th derivatives extend $\alpha$-Hölder continuous on $\partial \mathbb{D}$. This result, interesting on its own, allows us to obtain stability and regularities properties for families of complex geodesics (Section 2) and for their Lempert's projections, that is, the holomorphic retractions of $D$ with affine fibers onto complex geodesics introduced by Lempert in 21] which will play a fundamental role in our discussion (see Section 3).

The plan of the paper is as follows. In the first section we recall some preliminaries about complex geodesics, the boundary spherical representation of Chang, $\mathrm{Hu}$ and Lee [13] and the results in [11] as needed to make this work as self-contained as possible. In Section 2 we deal with regularity for families of complex geodesics by studying their differential properties and, as a corollary of our construction, we recover with a different proof some stability results by Huang [18, 19]. In the third section we study Lempert's projections. We first show that holomorphic retractions on given complex geodesics are not unique, but Lempert's projections can be characterized as the unique retractions with affine fibers. Then we examine the variation of Lempert's projections with respect to boundary data and prove regularity. In Section 4 we investigate the shape of horospheres. We prove that they are strongly convex away from their center (where they are $C^{1,1}$ ) using Jacobi vector fields. In the fifth section we state and prove the Phragmen-Lindelöf theorem for strongly convex domains and we compute the limits of $\Omega_{D, p}$ along non-complextangential directions. In the sixth section we prove (0.3) and in Section 7 we deal 
with uniqueness. Finally, in Section 8 we recall Demailly's theory for reproducing plurisubharmonic functions and find the explicit reproducing formulas using $\Omega_{D, p}$.

\section{Preliminaries}

Let $D$ be a bounded strongly convex domain in $\mathbb{C}^{n}$ with smooth boundary. A complex geodesic is a holomorphic map $\varphi: \mathbb{D} \rightarrow D$ which is an isometry between the Poincaré metric of $\mathbb{D}=\{\zeta \in \mathbb{C}:|\zeta|<1\}$ and the Kobayashi distance $k_{D}$ in $D$.

According to Lempert (see 21] and [1), any complex geodesic extends smoothly to the boundary of the disc and $\varphi(\partial \mathbb{D}) \subset \partial D$. Moreover, given any two points $z, w \in \bar{D}, z \neq w$, there exists a complex geodesic $\varphi: \mathbb{D} \rightarrow D$ such that $z, w \in \varphi(\overline{\mathbb{D}})$. Such a geodesic is unique up to pre-composition with automorphisms of $\mathbb{D}$. Also, if $z \in \bar{D}$ and $v \in \mathbb{C}^{n} \backslash\{O\}$ (and $v \notin T_{z} \partial D$ if $z \in \partial D$ ) there exists a unique (still, up to pre-composition with automorphisms of $\mathbb{D}$ ) complex geodesic $\varphi: \mathbb{D} \rightarrow D$ such that $z \in \varphi(\overline{\mathbb{D}})$ and $\varphi(\overline{\mathbb{D}})$ is parallel to $v$ (in the case $z, w \in \partial D$ this follows from Abate [3] and Chang, Hu and Lee [13]). In the cases $z \in D$ and $w \in \bar{D}, w \neq z$ (respectively $v \in T_{z} D$ ) one can uniquely choose a complex geodesic $\varphi: \mathbb{D} \rightarrow D$ requiring that $\varphi(0)=z$ and $\varphi(t)=w$ for some $0<t \leq 1$, with $t=1$ if and only if $w \in \partial D$ (respect. $\varphi^{\prime}(0)=t v$ for some $t>0$ ). With an abuse of notation, when no risk of confusion arises, we also call "complex geodesic" the image of a complex geodesic $\varphi: \mathbb{D} \rightarrow D$.

If $\varphi: \mathbb{D} \rightarrow D$ is a complex geodesic, then there exists a holomorphic map $\widetilde{\varphi}: \mathbb{D} \rightarrow \mathbb{C}^{n}$, called the dual map of $\varphi$, such that $\widetilde{\varphi}$ extends smoothly to $\partial \mathbb{D}$ and $\widetilde{\varphi}\left(e^{i \theta}\right)=e^{i \theta} \mu\left(e^{i \theta}\right) \partial r_{\varphi\left(e^{i \theta}\right)}$, with $r$ being a defining function of $D$ near $\varphi(\partial \mathbb{D})$ and $\mu>0$ normalized so that

$$
\widetilde{\varphi}(\zeta) \cdot \varphi^{\prime}(\zeta) \equiv 1
$$

for all $\zeta \in \mathbb{D}($ see $[21)$.

Let $\varphi: \mathbb{D} \rightarrow D$ be a complex geodesic. In 22] and [23] (see also Pang [26]) Lempert defines a biholomorphic change of coordinates $G: D \rightarrow D^{\prime}$ which "linearizes" $\varphi$. Namely, he proves that $G$ extends smoothly on $\partial D$, that $G \circ \varphi(\zeta)=(\zeta, 0, \ldots, 0)$ and that $\widetilde{G \circ \varphi}(\zeta)=(1,0, \ldots, 0)$. The domain $D^{\prime}=G(D)$ is no longer convex in general, but it is strictly linearly convex near $G(\varphi(\partial \mathbb{D}))$; namely, the real Hessian of any defining function of $D^{\prime}$ is positive on the complex tangent space at any point of $\partial D^{\prime}$ near $G(\varphi(\partial \mathbb{D}))$. In the rest of the paper we will refer to such a $G$ as the Lempert biholomorphism which linearizes $\varphi$.

Considering the foliation of all complex geodesics passing through a given point $z_{0} \in D$, Lempert constructed a map $\Phi_{z_{0}}: D \rightarrow \mathbb{B}^{n}$, called a spherical representation of $D$ at $z_{0}$, which is defined by $\Phi_{z_{0}}(z)=\zeta \varphi_{z}^{\prime}(0) /\left\|\varphi_{z}^{\prime}(0)\right\| \in \mathbb{B}^{n}$, where $\varphi_{z}: \mathbb{D} \rightarrow D$ is a complex geodesic such that $\varphi_{z}(0)=z_{0}, \varphi_{z}(\zeta)=z$ for $z \neq z_{0}$ and $\Phi_{z_{0}}\left(z_{0}\right)=O$. The map $\Phi_{z_{0}}$, which is continuous on $D$, extends $C^{\infty}$ on $\bar{D} \backslash\left\{z_{0}\right\}$. In his work 21] Lempert proved that $L_{D, z_{0}}:=\log \left\|\Phi_{z_{0}}\right\|$ solves (0.1).

Similarly, considering all complex geodesics whose closure contains a given boundary point $p \in \partial D$, Chang, Hu and Lee (see [13, Theorem 3]) constructed a boundary spherical representation. For the reader's convenience and since it will be useful later, we recall the construction of Chang, Hu and Lee as needed for our purpose. Let $p \in \partial D$ and let $\nu_{p}$ be the unit outward normal to $\partial D$ at $p$. Denote

$$
L_{p}:=\left\{v \in \mathbb{C}^{n} \mid\|v\|=1,\left\langle v, \nu_{p}\right\rangle>0, i v \in T_{p} \partial D\right\}
$$


and let $v \in L_{p}$. In what follows we will say that a complex geodesic $\varphi_{v}: \mathbb{D} \rightarrow D$ whose closure contains the point $p \in \partial D$ is in the Chang-Hu-Lee normal parametrization (with respect to $\left.v \in L_{p}\right)$ if $\varphi(1)=p$ and $\varphi^{\prime}(1)=\left\langle v, \overline{\nu_{p}}\right\rangle v$ and $\operatorname{Im}\left\langle\varphi^{\prime \prime}(1), \overline{\nu_{p}}\right\rangle=$ 0 . In [13] Chang, $\mathrm{Hu}$ and Lee proved that for all $v \in L_{p}$ there exists a unique complex geodesic in the Chang-Hu-Lee normal parametrization with respect to $v$.

Up to rigid movements of $\mathbb{C}^{n}$, assume that $\nu_{p}=e_{1}=(1,0, \ldots, 0)$, and thus $L_{p}$ reduces to $L_{p}=\left\{v=\left(v_{1}, \ldots, v_{n}\right) \in \mathbb{C}^{n}:\|v\|=1, v_{1}>0\right\}$. For any $v \in L_{p}$ the map $\eta_{v}: \mathbb{D} \ni \zeta \mapsto e_{1}+(\zeta-1) v_{1} v$ is a complex geodesic of $\mathbb{B}^{n}, \eta_{v}(1)=e_{1}$ and $\eta_{v}^{\prime}(1)=v_{1} v$. Then the boundary spherical representation $\Phi_{p}: D \rightarrow \mathbb{B}^{n}$ is defined as follows:

$$
\Phi_{p}(z)=e_{1}+\left(\zeta_{z}-1\right) v_{z, 1} v_{z},
$$

where $\zeta_{z} \in \mathbb{D}$ and $v_{z} \in L_{p}$ are the unique data such that $\varphi_{v_{z}}\left(\zeta_{z}\right)=z$. The map $\Phi_{p}$ is a smooth diffeomorphism whose inverse is $\Phi_{p}^{-1}(w)=\varphi_{v_{w}}\left(\zeta_{w}\right)$, where $\zeta_{w} \in \mathbb{D}$ and $v_{w} \in L_{p}$ are the unique data such that $w=\eta_{v_{w}}\left(\zeta_{w}\right)$. Moreover $\Phi_{p}, \Phi_{p}^{-1}$ extend continuously up to the boundary and $\Phi_{p}(p)=e_{1}$. In particular it follows that $\Phi_{p}$ is holomorphic on all complex geodesics in $D$ whose closure contains $p$ and sends such complex geodesics to complex geodesics in $\mathbb{B}^{n}$ whose closure contains $e_{1}$.

Following Abate ([1], [2]) we define a horosphere $E_{D}\left(p, z_{0}, R\right)$ of center $p \in \partial D$, pole $z_{0} \in D$ and radius $R>0$ as

$$
E_{D}\left(p, z_{0}, R\right):=\left\{z \in D: \lim _{w \rightarrow p}\left[k_{D}(z, w)-k_{D}\left(z_{0}, w\right)\right]<\frac{1}{2} \log R\right\} .
$$

The limit in the definition of $E_{D}\left(p, z_{0}, R\right)$ exists since $D$ is strongly convex and any such horosphere $E_{D}\left(p, z_{0}, R\right)$ is a sub-level set of the Busemann function of any geodesic whose closure contains $p$ (see [34]).

In [11, Corollary 6.2] it was proved that $\Phi_{p}$ maps horospheres of $D$ centered at $p$ onto horospheres of $\mathbb{B}^{n}$ centered at $e_{1}$, which, since horospheres of $\mathbb{B}^{n}$ are complex ellipsoid, implies in particular that the boundaries of horospheres are smooth away from the center $p$.

Let $\Omega_{\mathbb{B}^{n}, e_{1}}(z)=-\frac{1-\|z\|^{2}}{\left|1-z_{1}\right|^{2}}$. The sub-level sets of $\Omega_{\mathbb{B}^{n}, e_{1}}$ correspond to horospheres of $\mathbb{B}^{n}$ with center $e_{1}$ and pole $O$ (see, e.g., [1], 2]). In [11] we defined

$$
\Omega_{D, p}:=\Omega_{\mathbb{B}^{n}, e_{1}} \circ \Phi_{p}
$$

and proved Theorem 0.1. For further use we notice that

$$
E_{D}\left(p, \Phi_{p}^{-1}(O), R\right)=\left\{z \in D: \Omega_{D, p}(z)<-\frac{1}{R}\right\} .
$$

Finally, let

$$
P(\zeta):=\frac{1-|\zeta|^{2}}{|1-\zeta|^{2}}
$$

be the Poisson kernel on $\mathbb{D}=\{|\zeta|<1\}$. Recall that $P$ is harmonic in $\mathbb{D}, \lim _{\zeta \rightarrow x} P(\zeta)$ $=0$ for $x \in \partial \mathbb{D} \backslash\{1\}$ and $\lim _{\mathbb{R} \ni r \rightarrow 1^{-}} P(r)(1-r)=2$. From the very definition it follows that for all $v \in L_{p}$

$$
\Omega_{D, p}\left(\varphi_{v}(\zeta)\right)=-P(\zeta) / v_{1}^{2} .
$$




\section{Regularity FOR FAMILIES OF COMPLEX GEODESICS}

In this section we state some results about regularity of families of complex geodesics in strongly convex domains which we will need later.

From these we also rediscovered some facts already known or implicitly contained in other papers such as [21, 22], [18, [19]. Our presentation owes much to the works [16], 35], 33].

In this section $D$ will be a bounded strongly convex domain of $\mathbb{C}^{n}$ with smooth boundary. Given $k \geq 2$ and $\alpha \in(1 / 2,1)$ we denote by $\mathcal{O}^{k+\alpha}\left(\overline{\mathbb{D}}, \mathbb{C}^{n}\right)$ the set of all holomorphic maps from $\mathbb{D}$ to $\mathbb{C}^{N}$ which extends $C^{k}$ on $\overline{\mathbb{D}}$ and such that their $k$-th derivatives are $\alpha$-Hölder on $\overline{\mathbb{D}}$ (a map $f: \overline{\mathbb{D}} \rightarrow \mathbb{C}^{n}$ is $\alpha$-Hölder if there exists $C>0$ such that $\left\|f\left(\zeta_{0}\right)-f\left(\zeta_{1}\right)\right\| \leq C\left|\zeta_{0}-\zeta_{1}\right|^{\alpha}$ for all $\left.\zeta_{0}, \zeta_{1} \in \mathbb{D}\right)$. The set $\mathcal{O}^{k+\alpha}\left(\overline{\mathbb{D}}, \mathbb{C}^{n}\right)$ is a complex Banach space when endowed with the norm

$$
\|f\|_{k+\alpha}=\sum_{j=1}^{k} \sup _{\zeta \in \partial \mathbb{D}}\left\|f^{(j)}(\zeta)\right\|+\sup _{\zeta_{0}, \zeta_{1} \in \overline{\mathbb{D}}, \zeta_{0} \neq \zeta_{1}} \frac{\left\|f^{(k)}\left(\zeta_{0}\right)-f^{(k)}\left(\zeta_{1}\right)\right\|}{\left|\zeta_{0}-\zeta_{1}\right|^{\alpha}} .
$$

Let $\mathcal{G}$ be the set of complex geodesics from $\mathbb{D}$ to $D$. By Lempert's theory 21 it follows that $\mathcal{G} \subset \mathcal{O}^{k+\alpha}\left(\overline{\mathbb{D}}, \mathbb{C}^{n}\right)$. Let us also denote by $M \subset \mathcal{O}^{k+\alpha}\left(\overline{\mathbb{D}}, \mathbb{C}^{n}\right)$ the set of constants with value in $\partial D$. It is clear that $M$ is a closed set in $\mathcal{O}^{k+\alpha}\left(\overline{\mathbb{D}}, \mathbb{C}^{n}\right)$.

Theorem 2.1. The set $\mathcal{G}$ is a closed submanifold of $\mathcal{O}^{k+\alpha}\left(\overline{\mathbb{D}}, \mathbb{C}^{n}\right) \backslash M$ of real dimension $4 n-1$.

Proof. Let $\left\{f_{n}\right\} \subset \mathcal{G}$ and assume that $f_{n} \rightarrow f$ in $\mathcal{O}^{k+\alpha}\left(\overline{\mathbb{D}}, \mathbb{C}^{n}\right)$. Since the domain $D$ is strongly (pseudo)convex, either $f(\mathbb{D}) \subset D$-and from the continuity of $k_{D}$ it follows easily that $f \in \mathcal{G}$ as well -or $f \in M$. Thus $\mathcal{G}$ is closed in $\mathcal{O}^{k+\alpha}\left(\overline{\mathbb{D}}, \mathbb{C}^{n}\right) \backslash M$. Let $f_{0} \in \mathcal{G}$. We want to prove that $\mathcal{G}$ is a submanifold of $\mathcal{O}^{k+\alpha}\left(\overline{\mathbb{D}}, \mathbb{C}^{n}\right)$ near $f_{0}$.

Let $G: D \rightarrow D^{\prime}=G(D)$ be the Lempert biholomorphisms which linearizes $f_{0}$. Then $G \circ f_{0}(\zeta)=(\zeta, 0, \ldots, 0)$ and the dual map $\widetilde{G \circ f_{0}}(\zeta) \equiv(1,0, \ldots, 0)$. Notice that $G$ extends $C^{\infty}$ up to $\partial D$. Thus we can extend (arbitrarily) $\left.G\right|_{\partial D}$ to some $C^{\infty}$ map, denoted by $\tilde{G}$, from $\mathbb{C}^{n}$ to $\mathbb{C}^{n}$. We thus have a morphism $\Lambda: \mathcal{C}^{k+\alpha}\left(\partial \mathbb{D}, \mathbb{C}^{n}\right) \rightarrow \mathcal{C}^{k+\alpha}\left(\partial \mathbb{D}, \mathbb{C}^{n}\right)$ given by $\Lambda(f)=\tilde{G} \circ f$. The morphism $\Lambda$ is $C^{\infty}$ and maps the set of complex geodesics of $D$ onto the set of complex geodesics of $D^{\prime}$. Assume for the moment that we proved that $\Lambda(\mathcal{G})$ is a finite dimensional submanifold of $\mathcal{O}^{k+\alpha}\left(\overline{\mathbb{D}}, \mathbb{C}^{n}\right)$ near $G \circ f_{0}$, and thus a finite dimensional submanifold of $\mathcal{C}^{k+\alpha}\left(\partial \mathbb{D}, \mathbb{C}^{n}\right)$. Repeating the argument with $G^{-1}$, we find a $C^{\infty}$ map $\Lambda^{\prime}$ : $\mathcal{C}^{k+\alpha}\left(\partial \mathbb{D}, \mathbb{C}^{n}\right) \rightarrow \mathcal{C}^{k+\alpha}\left(\partial \mathbb{D}, \mathbb{C}^{n}\right)$ such that $\left.\Lambda \circ \Lambda^{\prime}\right|_{\Lambda(\mathcal{G})}=\left.\operatorname{Id}\right|_{\Lambda(\mathcal{G})}$. Thus $\left.\Lambda^{\prime}\right|_{\Lambda(\mathcal{G})}$ is an embedding with $d \Lambda^{\prime}(T \Lambda(\mathcal{G})$ ) finite dimensional, thus closed and complemented in $\mathcal{C}^{k+\alpha}\left(\partial \mathbb{D}, \mathbb{C}^{n}\right)$. Therefore $\mathcal{G}=\Lambda^{\prime}(\Lambda(\mathcal{G}))$ is a finite dimensional submanifold (see, e.g., [5] $)$. We are then left to show that $\Lambda(\mathcal{G})$ is a finite dimensional submanifold.

Thus, we can assume from the beginning that $f_{0}(\zeta)=(\zeta, 0, \ldots, 0)$ and $\tilde{f}_{0}(\zeta)=$ $(1,0, \ldots, 0)$ in $D$-here however the domain $D$ is no longer strongly convex, but it is strictly linearly convex near $f_{0}(\partial \mathbb{D})$. By the very definition of the dual map and by (1.1) it follows that if $f$ is a complex geodesic of $D$ close to $f_{0}$ in $\mathcal{O}^{k+\alpha}\left(\overline{\mathbb{D}}, \mathbb{C}^{n}\right)$, then $\tilde{f}$ is close to $\tilde{f}_{0}$ in $\mathcal{O}^{k+\alpha}\left(\overline{\mathbb{D}}, \mathbb{C}^{n}\right)$, where, with some abuse of notation, we identify the one form $\tilde{f}$ with the vector of its components.

Let $\mathbb{P}^{n-1}(\mathbb{C})$ be the space of complex hyperplanes passing through the origin $O$. Let $\Psi: \partial D \rightarrow \mathbb{C}^{n} \times \mathbb{P}^{n-1}(\mathbb{C})$ be defined by $\Psi(p):=\left(p, T_{p}^{\mathbb{C}} \partial D\right)$. Let $S=\Psi(\partial D)$. By the very definition $\left(f_{0},\left[\tilde{f}_{0}\right]\right)(\partial \mathbb{D}) \subset S$. Moreover, since $\partial D$ is strongly pseudoconvex 
near $f_{0}(\partial D)$, then $S$ is a compact maximal totally real submanifold of $\mathbb{C}^{n} \times \mathbb{P}^{n-1}(\mathbb{C})$ near $\Psi\left(f_{0}(\partial D)\right)$ (see [36]).

Let $\left(z_{1}, \ldots, z_{n}\right)$ be coordinates in $\mathbb{C}^{n}$ and let $\left[z_{1}: \ldots: z_{n}\right]$ be the corresponding homogeneous coordinates in $\mathbb{P}^{n-1}(\mathbb{C})$; that is, the point $\left[z_{1}: \ldots: z_{n}\right]$ corresponds to the hyperplane $\left\{v=\left(v_{1}, \ldots, v_{n}\right) \in \mathbb{C}^{n}: \sum_{j=1}^{n} v_{j} \cdot z_{j}=0\right\}$. Let $U_{1}:=\left\{[z] \in \mathbb{P}^{n-1}(\mathbb{C}): z_{1} \neq 0\right\}$ be the chart obtained by identifying $\mathbb{C}^{n-1}$ with $U_{1}$ via $\left(w_{1}, \ldots, w_{n-1}\right) \rightarrow\left[1: w_{1}: \ldots: w_{n-1}\right]$ and let $R: \mathbb{C}^{n} \times \mathbb{C}^{n-1} \rightarrow \mathbb{R}^{2 n-1}$ be a defining function for $S \cap \mathbb{C}^{n} \times U_{1}$ (such a defining function can be easily defined starting from a global defining function of $D$ in $\left.\mathbb{C}^{n}\right)$. Let us consider $\mathcal{Q}=\{F=$ $\left.(f, g) \in \mathcal{O}^{k+\alpha}\left(\overline{\mathbb{D}}, \mathbb{C}^{n} \times \mathbb{C}^{n-1}\right):\left.R(f, g)\right|_{\partial \mathbb{D}} \equiv 0\right\}$. In other words, $F=(f, g) \in \mathcal{Q}$ if and only if $(f,[1: g])(\partial \mathbb{D}) \subset S$. In particular $\left(f_{0}, 0\right) \in \mathcal{Q}$. Note that if $f$ is a complex geodesic close to $f_{0}$ with dual map $\tilde{f}=\left(\tilde{f}_{1}, \tilde{f}_{2}\right) \in \mathbb{C} \times \mathbb{C}^{n-1}$, then $\min _{\zeta \in \overline{\mathbb{D}}}\left|\tilde{f}_{1}(\zeta)\right|>0$ and therefore $\left(f, \tilde{f}_{2} / \tilde{f}_{1}\right) \in \mathcal{Q}$. Conversely, if $(f, g) \in \mathcal{Q}$ and $(f, g)$ is close to $\left(f_{0}, 0\right)$ in $\mathcal{O}^{k+\alpha}\left(\overline{\mathbb{D}}, \mathbb{C}^{n} \times \mathbb{C}^{n-1}\right)$, then $\left|f_{1}^{\prime}(\zeta)+\sum_{j=2}^{n} f_{j}^{\prime}(\zeta) g_{j}(\zeta)\right|>0$ for all $\zeta \in \overline{\mathbb{D}}$, and then $f$ is a stationary disc in $D$ with dual map $(1, g) /\left(f_{1}^{\prime}+\sum_{j=2}^{n} f_{j}^{\prime} g_{j}\right)$, that is, a complex geodesic. It should be remarked that in this argument one cannot refer directly to Lempert's theory because $D$ is not strongly convex in general. However, since $\partial D$ is strongly pseudoconvex near $f_{0}(\partial \mathbb{D})$, then for $f$ close to $f_{0}$ in $\mathcal{O}^{k+\alpha}\left(\overline{\mathbb{D}}, \mathbb{C}^{n}\right)$ one can use Pang's results to relate stationarity to extremality; see [26, Section 2].

The previous discussion shows that there exists an open neighborhood $W_{0} \subset$ $\mathcal{O}^{k+\alpha}\left(\overline{\mathbb{D}}, \mathbb{C}^{n} \times \mathbb{C}^{n-1}\right)$ of $\left(f_{0}, 0\right)$ such that $\pi_{1}: \mathcal{Q} \cap W_{0} \rightarrow \mathcal{G} \cap \pi_{1}\left(W_{0}\right)$ is bijective, where $\pi_{1}$ is the projection on the first factor, namely $\pi_{1}(f, g):=f$. The map $\left.\pi_{1}\right|_{\mathcal{Q} \cap W_{0}}$ is clearly $C^{\infty}$, and its inverse is $C^{\infty}$ as well, being given by $f \mapsto\left(f, \tilde{f}_{2} / \tilde{f}_{1}\right)$, with $\tilde{f}=\left(\tilde{f}_{1}, \tilde{f}_{2}\right) \in \mathbb{C} \times \mathbb{C}^{n-1}$ the dual of $f$. Thus $\left.d \pi_{1}\right|_{T \mathcal{Q}}$ is injective and its image is finite dimensional, and hence is closed and complemented in $\mathcal{O}^{k+\alpha}\left(\overline{\mathbb{D}}, \mathbb{C}^{n}\right)$. Therefore, if we prove that $\mathcal{Q}$ is a finite dimensional submanifold of $\mathcal{O}^{k+\alpha}\left(\overline{\mathbb{D}}, \mathbb{C}^{n} \times \mathbb{C}^{n-1}\right)$ near $\left(f_{0}, 0\right)$, then the claim on $\mathcal{G}$ will follow. To prove that $\mathcal{Q}$ is a submanifold by means of the implicit function theorem in Banach spaces, it is enough to show that $d R_{f_{0}}: \mathcal{O}^{k+\alpha}\left(\overline{\mathbb{D}}, \mathbb{C}^{n} \times \mathbb{C}^{n-1}\right) \rightarrow \mathcal{O}^{k+\alpha}\left(\overline{\mathbb{D}}, \mathbb{R}^{n}\right)$ is surjective and its kernel is complemented in $\mathcal{O}^{k+\alpha}\left(\overline{\mathbb{D}}, \mathbb{C}^{n} \times \mathbb{C}^{n-1}\right)$. We have $d R_{f_{0}}(f(\zeta))=2 \operatorname{Re} A f(\zeta)$, with $A$ being the $(2 n-1) \times(2 n-1)$ matrix with entries $\frac{\partial R_{j}}{\partial z_{k}}\left(f_{0}\right)$. Since $S$ is maximal totally real, arguing as in [32, Theorem 3.1, Lemma 3.2] one can prove that all the Birkhoff partial indices of the operator $f \mapsto 2 \operatorname{Re} A f(\zeta)$ are $\geq 1$, and thus by [16] (see also [35] and [33] $) d R_{f_{0}}$ is surjective. Notice that the computation of Birkhoff partial indices in [32, Lemma 3.2] was proved under the assumption that $\partial D$ is strongly convex. It is easy to check that in fact such a result holds for strictly linearly convex domains, and therefore it can be used here since, in Lempert's coordinates, the domain is strictly linearly convex near $f_{0}(\partial \mathbb{D})$. Finally, a direct computation (or see [34]) shows that its kernel has finite (real) dimension $4 n-1$, and therefore $\mathcal{Q}$ is a submanifold of dimension $4 n-1$ near $f_{0}$.

Let $\kappa_{D}$ be the Kobayashi metric in $D$. According to Lempert ([21], 23]) the $\operatorname{map} D \times\left(\mathbb{C}^{n} \backslash\{O\}\right) \rightarrow \mathbb{R}$ given by $(z, v) \mapsto \kappa_{D}(z ; v)$ is $C^{\infty}$. Moreover, since $\kappa_{D}(z, \lambda v)=\lambda \kappa_{D}(z, v)$ for all $(z, v) \in D \times\left(\mathbb{C}^{n} \backslash\{O\}\right)$ and $\lambda>0$, it follows that $d\left(\kappa_{D}\right)_{(z, v)} \neq 0$ for all $(z, v) \in D \times\left(\mathbb{C}^{n} \backslash\{O\}\right)$. Therefore the set

$$
\mathcal{K}=\left\{(z, v) \in D \times\left(\mathbb{C}^{n} \backslash\{O\}\right): \kappa_{D}(z ; v)=1\right\}
$$

is a $(4 n-1)$-real dimensional submanifold of $D \times\left(\mathbb{C}^{n} \backslash\{O\}\right)$. 
Theorem 2.2. The map $V: \mathcal{G} \rightarrow \mathcal{K}$ defined by $V: f \mapsto\left(f(0), f^{\prime}(0)\right)$ is a diffeomorphism.

Proof. By the uniqueness of complex geodesics 21, the map $V$ is bijective. Since $V$ is the restriction of a linear bounded map from $\mathcal{O}^{k+\alpha}\left(\overline{\mathbb{D}}, \mathbb{C}^{n}\right)$ to $\mathbb{C}^{2 n}$, then it is linear and $C^{\infty}$. By [23, Theorem 5] the inverse $V^{-1}$ is $C^{\infty}$ as well, and hence $V$ is a diffeomorphism.

From this result we obtain some corollaries which will be useful later on.

Corollary 2.3. Let $\left\{f_{n}\right\} \subset \mathcal{G}$ be such that $f_{n} \rightarrow f$ uniformly on compacta of $\mathbb{D}$. If $f$ is not constant, then $f \in \mathcal{G}$ and $f_{n}^{(j)} \rightarrow f^{(j)}$ uniformly on $\overline{\mathbb{D}}$ for all $j=0,1, \ldots$

Proof. By Theorem 2.1 if $f$ is not constant, then it belongs to $\mathcal{G}$. Thus, $f_{n} \rightarrow f$ uniformly on compacta of $\mathbb{D}$ implies that $f_{n}(0) \rightarrow f(0)$ and $f_{n}^{\prime}(0) \rightarrow f^{\prime}(0)$. By Theorem 2.2 it follows that $f_{n} \rightarrow f$ in $\mathcal{O}^{k+\alpha}\left(\overline{\mathbb{D}}, \mathbb{C}^{n}\right)$ for all fixed $k \in \mathbb{N}$. In particular $f_{n}^{(j)} \rightarrow f^{(j)}$ for all $j=0,1, \ldots$

Corollary 2.4. If $(0,1) \ni t \mapsto f_{t} \in \mathcal{G}$ is a family of complex geodesics such that $t \mapsto f_{t}(0)$ and $t \mapsto f_{t}^{\prime}(0)$ are $C^{\infty}$, then $t \mapsto f_{t}$ is $C^{\infty}$ in $\mathcal{O}^{k+\alpha}\left(\overline{\mathbb{D}}, \mathbb{C}^{n}\right)$. In particular the map $\zeta \mapsto \frac{\partial^{j} f_{t}}{\partial t^{j}}(\zeta)$ is smooth on $\overline{\mathbb{D}}$ for all $j=1,2, \ldots$.

Lemma 2.5. The map $\mathcal{G} \ni f \mapsto f(0) \in D$ is proper.

Proof. If $\left\{z_{n}\right\} \subset D$ is such that $z_{n} \rightarrow z \in D$, let $f_{n} \in \mathcal{G}$ be such that $f_{n}(0)=z_{n}$. Let $\left\{f_{n_{k}}\right\}$ be a converging subsequence. Since the Kobayashi distance is continuous on $D$, it follows that the limit $f$ of $\left\{f_{n_{k}}\right\}$ is not constant. Then by Corollary 2.3 it follows that $f \in \mathcal{G}$ and $f(0)=z$. Hence the map $f \mapsto f(0)$ is proper.

As a straightforward corollary of Lemma 2.5 we have the following result, first proved using different methods by Huang [19, Proposition 1]:

Proposition 2.6. Let $c>0$ and let $\mathcal{G}_{c}:=\{f \in \mathcal{G}: \operatorname{dist}(f(0), \partial D) \geq c\}$. Then there exists $c^{\prime}>0$ such that $\|f\|_{k+\alpha} \leq c^{\prime}$ for every $f \in \mathcal{G}_{c}$.

\section{LEMPERT'S PROJECTIONS}

Let $D \subset \mathbb{C}^{n}$ be a bounded strongly convex domain with smooth boundary and let $\varphi: \mathbb{D} \rightarrow D$ be a complex geodesic. According to Lempert (21], 22], 23]), for all $z \in D$ the equation $\widetilde{\varphi}(\zeta) \cdot(z-\varphi(\zeta)) \equiv 0$ in the unknown $\zeta \in \mathbb{D}$ has a unique solution $\zeta:=\tilde{\rho}(z)$. The map $\tilde{\rho}: D \rightarrow \mathbb{D}$ is holomorphic, extends smoothly on $\partial D$ and is called the left inverse of $\varphi$ because it satisfies $\tilde{\rho} \circ \varphi=\mathrm{id}_{\mathbb{D}}$. By the very definition

$$
\widetilde{\varphi}(\tilde{\rho}(z)) \cdot(z-\varphi(\tilde{\rho}(z))) \equiv 0 .
$$

Remark 3.1. Let $z \in \bar{D}$. If $\zeta \in \overline{\mathbb{D}}$ is such that $\widetilde{\varphi}(\zeta) \cdot(z-\varphi(\zeta))=0$, then $\widetilde{\rho}(z)=\zeta$. Indeed, by the strong convexity of $\partial D$, if $z \in \bar{D} \backslash \varphi(\partial \mathbb{D})$, then the winding number of the function $\partial \mathbb{D} \ni \zeta \mapsto \widetilde{\varphi}(\zeta) \cdot(z-\varphi(\zeta))$ is 1 (see [22, 23]), hence $\zeta=\widetilde{\rho}(z)$. On the other hand, if $z=\varphi\left(e^{i t}\right)$ for some $t \in \mathbb{R}$, by continuity of $\widetilde{\rho}$ it follows that $\widetilde{\rho}\left(\varphi\left(e^{i t}\right)\right)=e^{i t}$. Suppose by contradiction that $\widetilde{\varphi}(\zeta) \cdot\left(\varphi\left(e^{i t}\right)-\varphi(\zeta)\right)=0$ for some $\zeta \in \overline{\mathbb{D}} \backslash\left\{e^{i t}\right\}$. Since the domain is strongly convex the interior of the real segment $\ell$ joining $\varphi\left(e^{i t}\right)$ to $\varphi(\zeta)$ is contained in $D$. Then the segment $\ell$ belongs to the fiber of $\widetilde{\rho}$ at $\varphi(\zeta)$ and, since $\widetilde{\rho}$ is continuous on $\bar{D}$, it follows that $\widetilde{\rho}\left(\varphi\left(e^{i t}\right)\right)=\zeta$ which contradicts $\widetilde{\rho}\left(\varphi\left(e^{i t}\right)\right)=e^{i t}$. 
Let $\varphi$ be a complex geodesic and let $\tilde{\rho}$ be its left-inverse. The map $\rho: D \rightarrow$ $\varphi(\mathbb{D}) \subset D$ defined as $\rho:=\varphi \circ \tilde{\rho}$ is a holomorphic retraction on $\varphi(\mathbb{D})$, i.e., $\rho$ is a holomorphic self-map of $D$ such that $\rho \circ \rho=\rho$ and $\rho(z)=z$ for any $z \in \varphi(\mathbb{D})$. It extends smoothly to $\partial D$ and is called the Lempert projection associated to $\varphi$. The triple $(\varphi, \rho, \tilde{\rho})$ is the so-called Lempert projection device. As remarked for instance in [10, p. 145] the Lempert projection $\rho$ depends only on the image $\varphi(\mathbb{D})$.

In this section we study regularity of Lempert's left-inverse. Before that, we make some comments on holomorphic retractions on strongly convex domains. We start with an example which shows that there exist infinitely many holomorphic retractions:

Example 3.2. Let $f_{j k}: \mathbb{B}^{n} \rightarrow \mathbb{D}$ be holomorphic functions, $j, k=2, \ldots, n$, and let $\epsilon<1 / 2 n$. The holomorphic map

$$
\rho(z):=\left(z_{1}+\epsilon \sum_{j, k=2}^{n} z_{j} z_{k} f_{j k}(z), 0, \ldots, 0\right)
$$

is a holomorphic retraction of $\mathbb{B}^{n}$ onto the complex geodesic $\varphi(\zeta)=(\zeta, 0, \ldots, 0)$. Indeed, it is clear that $\rho\left(\mathbb{B}^{n}\right) \subset \mathbb{C} \times\{(0, \ldots, 0)\}$, that $\rho^{2}=\rho$ and that $\rho$ is holomorphic. Moreover, if we let $r=\left|z_{1}\right|$, then $\left|z_{j}\right| \leq \sqrt{1-r^{2}}$ and $\left|z_{1}+\epsilon \sum_{j, k=2}^{n} z_{j} z_{k} f_{j k}(z)\right| \leq$ $r+n \epsilon\left(1-r^{2}\right)$, proving that for $\epsilon<1 / 2 n$ the image $\rho\left(\mathbb{B}^{n}\right) \subset \mathbb{B}^{n}$.

From (3.1) it follows that the fibers of Lempert's projection are intersections of $D$ with complex affine hyperplanes. Lempert's projection can be characterized exactly by this property:

Proposition 3.3. Let $\varphi: \mathbb{D} \rightarrow D$ be a complex geodesic. If $\rho: D \rightarrow \varphi(\mathbb{D})$ is a holomorphic retraction whose fibers are intersections of $D$ with complex affine hyperplanes, then $\rho$ is the Lempert projection. In other words, the Lempert projection is the only "affine-linear" retraction.

Proof. Let $\rho: D \rightarrow \varphi(\mathbb{D})$ be a retraction whose fibers are intersections of $D$ with complex affine hyperplanes. Let $E_{D}=E_{D}\left(\varphi\left(e^{i t}\right), \varphi\left(\zeta_{0}\right), R\right)$ be a horosphere of $D$ with radius $R>0$. Since $\rho \circ \varphi=\varphi$, if $z \in E_{D}$ we have

$$
\begin{aligned}
\lim _{w \rightarrow \varphi\left(e^{i t}\right)}\left[k_{D}(\rho(z), w)\right. & \left.-k_{D}\left(\varphi\left(\zeta_{0}\right), w\right)\right] \\
& =\lim _{r \rightarrow 1}\left[k_{D}\left(\rho(z), \rho\left(\varphi\left(r e^{i t}\right)\right)\right)-k_{D}\left(\varphi(0), \varphi\left(r e^{i t}\right)\right)\right] \\
& \leq \lim _{r \rightarrow 1}\left[k_{D}\left(z, \varphi\left(r e^{i t}\right)\right)-k_{D}\left(\varphi(0), \varphi\left(r e^{i t}\right)\right)\right]<\frac{1}{2} \log R .
\end{aligned}
$$

Therefore $\rho(z) \in E_{D} \cap \varphi(\mathbb{D})$. Let $\eta \in \mathbb{D}$ and $\varphi(\eta) \in \partial E_{D}$. Let $H$ be the affine hyperplane which contains $\rho^{-1}(\varphi(\eta))$. Then $E_{D} \cap H=\emptyset$, because if $z \in E_{D} \cap H$, then $\varphi(\eta)=\rho(z) \in E_{D} \cap \varphi(\mathbb{D})$, which is a contradiction. Since $\varphi(\eta) \in \overline{E_{D}} \cap H$ and $E_{D}$ is convex, it follows that $H-\varphi(\eta)=T_{\varphi(\eta)}^{\mathbb{C}} \partial E_{D}$. Now, $T_{\varphi(\eta)}^{\mathbb{C}} \partial E_{D}=\operatorname{ker}\left(\partial \rho_{L}\right)_{\varphi(\eta)}$, where $\rho_{L}$ is the Lempert projection. Thus $\rho$ and $\rho_{L}$ have the same fibers at $\varphi(\eta)$, and by the arbitrariness of the choices it follows that $\rho=\rho_{L}$ as claimed.

Next we examine the variation of the left inverse of Lempert's projection with respect to boundary data.

Lemma 3.4. Let $\left\{z_{k}\right\} \subset D$ be a sequence converging non-tangentially to $p$. Let $v_{k} \in L_{p}$ be such that $z_{k} \in \varphi_{v_{k}}(\mathbb{D})$ (where, for $v \in L_{p}, \varphi_{v}: \mathbb{D} \rightarrow D$ denotes the 
unique complex geodesic in the Chang-Hu-Lee normal parametrization with respect to $v)$. If $v_{k} \rightarrow v_{0}$, then $v_{0} \in L_{p}$ and $\varphi_{v_{k}} \rightarrow \varphi_{v_{0}}, \varphi_{v_{k}}^{(j)} \rightarrow \varphi_{v_{0}}^{(j)}$ uniformly on $\overline{\mathbb{D}}$ for all $j=1,2, \ldots$.

Proof. We can assume that $\nu_{p}=e_{1}$. To see that $v_{0} \in L_{p}$ we need to show that $\left\langle v_{0}, e_{1}\right\rangle>0$. Assume this is not the case. Then $v_{0} \in T_{p}^{\mathbb{C}} \partial \mathbb{D}$.

First of all, we claim that for any open neighborhood $U$ of $p$ it follows that $\varphi_{v_{k}}(\overline{\mathbb{D}}) \subset U$ eventually. Indeed, let $\Phi_{p}: D \rightarrow \mathbb{B}^{n}$ be the spherical representation of Chang-Hu-Lee and denote it by $\eta_{v_{k}}:=\Phi_{p} \circ \varphi_{v_{k}}$. By construction $\eta_{v_{k}}(\zeta)=$ $e_{1}+(\zeta-1)\left\langle v_{k}, e_{1}\right\rangle v_{k}$ and thus $\eta_{v_{k}}(\overline{\mathbb{D}}) \rightarrow e_{1}$. Since $\Phi_{p}^{-1}$ is uniformly continuous on $\overline{\mathbb{B}^{n}}$ the claim follows.

Therefore, $\left\{\varphi_{v_{k}}(\overline{\mathbb{D}})\right\}$ converges to $\{p\}$ and, by [18, Theorem 2], given any $\epsilon>0$ there exists $k_{0}$ such that, for all $k>k_{0}$, it follows that $\left\|\left(\varphi_{v_{k}}^{\prime}(\zeta)\right)_{N}\right\| \leq \epsilon\left\|\left(\varphi_{v_{k}}^{\prime}(\zeta)\right)_{T}\right\|$ for all $\zeta \in \mathbb{D}$ where, if $z \in D$ and $z^{\prime} \in \partial D$ is the unique point of $\partial D$ nearest to $z$, then, for all vectors $w \in T_{p} D=\mathbb{C}^{n}$ the vectors $w_{N}$ and $w_{T}$ denote the complex normal and the complex tangential components of $w$ at $z^{\prime}$ (namely, $w_{T} \in T_{z^{\prime}}^{\mathbb{C}} \partial D$ and $w_{N}=\left\langle w, \overline{\nu_{z^{\prime}}}\right\rangle \nu_{z^{\prime}}$, with $\nu_{z^{\prime}}$ being the unit outward normal to $\partial D$ at $\left.z^{\prime}\right)$.

Let $K \subset D$ be a cone with vertex $p$ such that $\left\{z_{k}\right\} \subset K$. In particular, there exists $c>0$ such that if $w \in \mathbb{C}^{n}$ and $(w-p) \in K$, then $\|w-p\|_{N} \geq c\|w-p\|_{T}$ (at $p$ ). Therefore, if $\gamma:[0,1] \rightarrow D \cup\{p\}$ is a $C^{\infty}$ curve such that $\gamma^{\prime}(1)=p$ and $\left\|\gamma^{\prime}(1)_{N}\right\| \leq(c / 2)\left\|\gamma^{\prime}(1)_{T}\right\|($ at $p)$, then $\gamma(t) \notin K$ for $t \approx 1$. Moreover, we can find a small open neighborhood $U$ of $p$ such that, if $\gamma([0,1)) \subset U \cap D$ and $\left\|\gamma^{\prime}(t)_{N}\right\| \leq(c / 2)\left\|\gamma^{\prime}(t)_{T}\right\|$ for all $t \in[0,1]$ (here the projection is at the point of $\partial D$ nearest to $\gamma(t))$, then $\gamma(t) \notin K$ for $t \in[0,1))$.

Now, let $k$ be such that $\varphi_{v_{k}}(\mathbb{D}) \subset U \cap D$ and $\left\|\left(\varphi_{v_{k}}^{\prime}(\zeta)\right)_{N}\right\| \leq(c / 2)\left\|\left(\varphi_{v_{k}}^{\prime}(\zeta)\right)_{T}\right\|$ for all $\zeta \in \mathbb{D}$. Let $\theta_{k} \in \operatorname{Aut}(\mathbb{D})$ be an automorphism such that $\theta_{k}(1)=1$ and $\varphi_{v_{k}}\left(\theta_{k}(0)\right)=z_{k}$. By the previous argument $\gamma(t):=\varphi_{v_{k}}\left(\theta_{k}(t)\right)$ does not belong to $K$ for any $t$, which contradicts the fact that $z_{k} \in K$. Thus $\left\langle v_{0}, e_{1}\right\rangle>0$ and $v_{0} \in L_{p}$.

We are left to show that $\varphi_{v_{k}} \rightarrow \varphi_{v_{0}}$ and $\varphi_{v_{k}}^{(j)} \rightarrow \varphi_{v_{0}}^{(j)}$ uniformly on $\overline{\mathbb{D}}$. Let $\eta_{v_{k}}:=\Phi_{p} \circ \varphi_{v_{k}}: \mathbb{D} \rightarrow \mathbb{B}^{n}$. By the very definition $\eta_{v_{k}}(\zeta)=e_{1}+(\zeta-1)\left\langle v_{k}, e_{1}\right\rangle v_{k}$ and clearly $\eta_{v_{k}} \rightarrow \eta_{v_{0}}$ uniformly on $\overline{\mathbb{D}}$. Since $\Phi_{p}$ is a homeomorphism between $\bar{D}$ and $\overline{\mathbb{B}^{n}}$ it follows that $\varphi_{v_{k}} \rightarrow \varphi_{v_{0}}$ uniformly on $\overline{\mathbb{D}}$. By Corollary 2.3 then $\varphi_{v_{k}}^{(j)} \rightarrow \varphi_{v_{0}}^{(j)}$ uniformly on $\overline{\mathbb{D}}$.

Lemma 3.5. For any $v \in L_{p}$ denote by $\varphi_{v}: \mathbb{D} \rightarrow D$ the unique complex geodesic in the Chang-Hu-Lee normal parametrization with respect to $v$ and let $\widetilde{\rho}_{v}$ be its leftinverse. Then, if $\left\{v_{k}\right\} \subset L_{p}$ is such that $v_{k} \rightarrow v_{0} \in L_{p}$ it follows that $d \widetilde{\rho}_{v_{k}} \rightarrow d \widetilde{\rho}_{v_{0}}$ uniformly on $\bar{D}$.

Proof. Differentiating (3.1) with respect to $z_{j}$ we obtain for $z \in \bar{D}$

$$
\frac{\partial \widetilde{\rho}_{v}}{\partial z_{j}} \widetilde{\varphi}_{v}^{\prime}\left(\widetilde{\rho}_{v}(z)\right) \cdot\left(z-\varphi_{v}\left(\widetilde{\rho}_{v}(z)\right)\right)+\widetilde{\varphi}_{v}\left(\widetilde{\rho}_{v}(z)\right) \cdot\left(e_{j}-\frac{\partial \widetilde{\rho}_{v}}{\partial z_{j}} \varphi_{v}^{\prime}\left(\widetilde{\rho}_{v}(z)\right)\right) \equiv 0
$$

holding for $z \in \bar{D}$. Taking into account that $\widetilde{\varphi}(\zeta) \cdot \varphi^{\prime}(\zeta) \equiv 1$, we have

$$
\frac{\partial \widetilde{\rho}_{v}}{\partial z_{j}}\left[\widetilde{\varphi}_{v}^{\prime}\left(\widetilde{\rho}_{v}(z)\right) \cdot\left(z-\varphi_{v}\left(\widetilde{\rho}_{v}(z)\right)\right)-1\right] \equiv-\widetilde{\varphi}_{v}\left(\widetilde{\rho}_{v}(z)\right) \cdot e_{j}
$$


Notice that, since $\widetilde{\varphi}_{v}(\zeta) \neq 0$ for all $\zeta \in \overline{\mathbb{D}}$, for all $z \in \bar{D}$ there exists $j \in\{1, \ldots, n\}$ such that $\widetilde{\varphi}_{v}\left(\widetilde{\rho}_{v}(z)\right) \cdot e_{j} \neq 0$. In particular it follows that

$$
\widetilde{\varphi}_{v}^{\prime}\left(\widetilde{\rho}_{v}(z)\right) \cdot\left(z-\varphi_{v}\left(\widetilde{\rho}_{v}(z)\right)\right)-1 \neq 0
$$

for all $z \in \bar{D}$. Therefore

$$
\frac{\partial \widetilde{\rho}_{v}}{\partial z_{j}}(z)=\frac{-\widetilde{\varphi}_{v}\left(\widetilde{\rho}_{v}(z)\right) \cdot e_{j}}{\widetilde{\varphi}_{v}^{\prime}\left(\widetilde{\rho}_{v}(z)\right) \cdot\left(z-\varphi_{v}\left(\widetilde{\rho}_{v}(z)\right)\right)-1} .
$$

Let $\left\{v_{k}\right\} \subset L_{p}$ be such that $v_{k} \rightarrow v_{0} \in L_{p}$. We claim that

$$
\tilde{\rho}_{v_{k}} \rightarrow \tilde{\rho}_{v_{0}}, \quad \widetilde{\varphi}_{v_{k}} \rightarrow \widetilde{\varphi}_{v_{0}}, \quad \widetilde{\varphi}_{v_{k}}^{\prime} \rightarrow \widetilde{\varphi}_{v_{0}}^{\prime} \quad \varphi_{v_{k}} \rightarrow \varphi_{v_{0}}
$$

uniformly on $\bar{D}$ and $\overline{\mathbb{D}}$ respectively. By Lemma 3.4 it follows that $\varphi_{v_{k}} \rightarrow \varphi_{v_{0}}$ uniformly on $\overline{\mathbb{D}}$.

As for $\widetilde{\varphi}_{v}$, if $v_{k} \rightarrow v_{0}$ in $L_{p}$ then by Lemma 3.4 it follows that $\varphi_{v_{k}}^{(j)} \rightarrow \varphi_{v_{0}}^{(j)}$ uniformly on $\overline{\mathbb{D}}$ for all $j=0,1,2, \ldots$. By the very definition and by (1.1), if $r$ is a defining function for $\partial D$, it follows that for $\zeta \in \partial \mathbb{D}$

$$
\widetilde{\varphi}_{v_{k}}(\zeta)=\frac{1}{\partial r_{\varphi_{v_{k}}(\zeta)}\left(\varphi_{v_{k}}^{\prime}(\zeta)\right)} \partial r_{\varphi_{v_{k}}(\zeta)},
$$

and therefore, since $\left|\partial r_{\varphi_{v_{k}}(\zeta)}\left(\varphi_{v_{k}}^{\prime}(\zeta)\right)\right| \geq c>0$ for all $k$, it follows that $\widetilde{\varphi}_{v_{k}} \rightarrow$ $\widetilde{\varphi}_{v_{0}}$ uniformly on $\partial \mathbb{D}$. By the maximum principle $\widetilde{\varphi}_{v_{k}} \rightarrow \widetilde{\varphi}_{v_{0}}$ uniformly on $\overline{\mathbb{D}}$. Differentiating (3.6) for $\zeta=e^{i t}$ and $t \in \mathbb{R}$ by $\frac{d}{d t}$ we see that $\widetilde{\varphi}_{v_{k}}^{\prime}$ is expressed as a continuous combination of $\varphi_{v_{k}}, \varphi_{v_{k}}^{\prime}, \varphi_{v_{k}}^{\prime \prime}$, and by Lemma 3.4 it follows then that $\widetilde{\varphi}_{v_{k}}^{\prime} \rightarrow \widetilde{\varphi}_{v_{0}}^{\prime}$ uniformly on $\overline{\mathbb{D}}$.

We are left to show that $\widetilde{\rho}_{v_{k}} \rightarrow \widetilde{\rho}_{v_{0}}$ uniformly on $\bar{D}$. If not, there exists a sequence $\left\{z_{k_{m}}\right\} \subset \bar{D}$ (which we may assume converging to some $z_{0} \in \bar{D}$ ) such that $\left|\widetilde{\rho}_{v_{k_{m}}}\left(z_{k_{m}}\right)-\widetilde{\rho}_{v_{0}}\left(z_{k_{m}}\right)\right|>\epsilon_{0}$ for some $\epsilon_{0}>0$ and for all $k_{m}$. By (3.1) it follows that for all $k_{m}$

$$
\widetilde{\varphi}_{v_{k_{m}}}\left(\widetilde{\rho}_{v_{k_{m}}}\left(z_{k_{m}}\right)\right) \cdot\left(z_{k_{m}}-\varphi_{v_{k_{m}}}\left(\widetilde{\rho}_{v_{k_{m}}}\left(z_{k_{m}}\right)\right)\right)=0 .
$$

Up to subsequences, we can assume that $\widetilde{\rho}_{v_{k_{m}}}\left(z_{k_{m}}\right) \rightarrow \zeta_{0} \in \overline{\mathbb{D}}$. For what we have already proved it then follows that

$$
\widetilde{\varphi}_{v_{0}}\left(\zeta_{0}\right) \cdot\left(z_{0}-\varphi_{v_{0}}\left(\zeta_{0}\right)\right)=0 .
$$

This implies that $\zeta_{0}=\widetilde{\rho}_{v_{0}}\left(z_{0}\right)$, since the only zero of the function $\zeta \mapsto \widetilde{\varphi}_{v_{0}}(\zeta)$. $\left(z_{0}-\varphi_{v_{0}}(\zeta)\right)$ is $\widetilde{\rho}_{v_{0}}\left(z_{0}\right)$ by Remark 3.1. But then both $\left\{\widetilde{\rho}_{v_{k_{m}}}\left(z_{k_{m}}\right)\right\}$ and $\left\{\widetilde{\rho}_{v_{0}}\left(z_{k_{m}}\right)\right\}$ converge to $\widetilde{\rho}_{v_{0}}\left(z_{0}\right)$, and then $\left|\widetilde{\rho}_{v_{k_{m}}}\left(z_{k_{m}}\right)-\widetilde{\rho}_{v_{0}}\left(z_{k_{m}}\right)\right| \rightarrow 0$, contradiction. Thus $\widetilde{\rho}_{v_{k}} \rightarrow \widetilde{\rho}_{v_{0}}$ uniformly on $\bar{D}$, and the claim is proved.

Since, as we remarked at the beginning, the denominator of the right hand side of (3.5) for $v=v_{0}$ is never zero for all $z \in \bar{D}$, the previous claim implies that $d \widetilde{\rho}_{v_{k}} \rightarrow d \widetilde{\rho}_{v_{0}}$ uniformly on $\bar{D}$.

Remark 3.6. By (3.5) it follows that $d\left(\widetilde{\rho}_{v}\right)_{p}=\widetilde{\varphi}(1)$ and by (3.6) we have (cf. 1, Lemma 2.6.44]) for $w \in \mathbb{C}^{n}$

$$
d\left(\widetilde{\rho}_{v}\right)_{p}(w)=\frac{\partial r_{p}(w)}{\partial r_{p}\left(\varphi_{v}^{\prime}(1)\right)}=\frac{\left\langle w, \nu_{p}\right\rangle}{\left\langle\varphi_{v}^{\prime}(1), \nu_{p}\right\rangle} .
$$




\section{The ShAPE OF HOROSPHERES}

Let $D \subset \mathbb{C}^{n}$ be a bounded strongly convex domain and let $p \in \partial D$. As we recalled in Section 1, for any $R>0$ and $z_{0} \in D$, the set $\partial E_{D}\left(p, z_{0}, R\right)$ is smooth away from its center $p \in \partial D$. It should be noted that smoothness of horospheres away from the center was known after [34, Section 4], but we do not know any previous reference for this fact.

In [1] (see also 4]) it is proved that horospheres are convex domains (since they are an increasing union of Kobayashi balls of $D$ ). In [11, Remark 4.2], referring to [1. Corollary 2.6.49] it was claimed that (boundaries of) horospheres are strongly convex at their center. Unfortunately the proof of [1, Corollary 2.6.49] does not seem to show smoothness at the center, and thus one can only infer that horospheres are geometrically strictly convex (i.e., the intersection of their closure with the supporting hyperplane at the center is just the center). However from [2, pp. 231232] it follows that if $E_{D}\left(p, z_{0}, R\right) \subset D$ is a horosphere of center $p \in \partial D$ and radius $R>0$ and $\mathbb{B} \subset D$ is a ball tangent to $\partial D$ at $p$, then there exists a horosphere $E_{\mathbb{B}}\left(p, R^{\prime}\right) \subset \mathbb{B}$ for some $R^{\prime}>0$ such that $E_{\mathbb{B}}\left(p, R^{\prime}\right) \subset E_{D}\left(p, z_{0}, R\right)$. In particular, since horospheres of the ball $\mathbb{B}$ are smooth complex ellipsoids, it follows that there exists a ball $\mathbb{B}^{\prime} \subset E_{D}\left(p, z_{0}, R\right)$ tangent to $\partial E_{D}\left(p, z_{0}, R\right)$ at $p$. Namely, horospheres have the inner-ball property at the center. Therefore $\partial E_{D}\left(p, z_{0}, R\right)$ is $C^{1,1}$ at $p$ (see, e.g., [17, Proposition 2.4.3]).

Here we prove that the boundaries of horospheres are strongly convex away from the center:

Theorem 4.1. Let $D \subset \mathbb{C}^{n}$ be a strongly convex domain with smooth boundary. Let $p \in \partial D$. Let $E_{D}(p, R)$ be a horosphere in $D$ with center $p$ and radius $R>0$. The boundary $\partial E_{D}(p, R) \backslash\{p\}$ is smooth and strongly convex.

Proof. Let $\Omega_{D, p}$ be the function defined in Theorem 0.1 . Its level sets are boundaries of horospheres of $D$ with center $p$. Thus, to show that such boundaries are strongly convex we need to prove that the (real) Hessian of $\Omega_{D, p}$ is positive definite on the tangent space of $\partial E_{D}(p, R)$ (for all $R>0$ ). It is known (see [1]) that $\partial E_{D}(p, R)$ are convex for all $R>0$ (and strongly pseudoconvex for all $R>0$ and strongly convex for big radii; see [11, Remark 7]). Thus the real Hessian of $\Omega_{D, p}$ is non-negative definite on the (real) tangent space of $\partial E_{D}(p, R)$ for all $R>0$.

Let $q \in D$ and let $\varphi: \mathbb{D} \rightarrow D$ be a complex geodesic such that $\varphi(0)=q$ and $\varphi(1)=p$. Up to post-composing with automorphisms of $\mathbb{B}^{n}$ fixing $e_{1}$, we can suppose that $\Phi_{p}(q)=O$. Thus $\Phi_{p}(\varphi(\zeta))=(\zeta, O)$. Let $F: D \rightarrow \mathbb{H}^{n}:=\{(\zeta, w) \in$ $\left.\mathbb{C} \times \mathbb{C}^{n-1}: \operatorname{Im} \zeta>\|w\|^{2}\right\}$ be given by $F=C \circ \Phi_{p}$, where $C: \mathbb{B}^{n} \rightarrow \mathbb{H}^{n}$ is the Cayley transform defined as

$$
C(\zeta, w)=\left(i \frac{1+\zeta}{1-\zeta}, \frac{w}{1-\zeta}\right), \quad(\zeta, w) \in \mathbb{C} \times \mathbb{C}^{n-1}
$$

We write $F(z)=\left(F_{0}(z), \tilde{F}(z)\right) \in \mathbb{C} \times \mathbb{C}^{n-1}$. By definition,

$$
F_{0}(\varphi(\zeta))=i \frac{1+\zeta}{1-\zeta}, \quad \tilde{F}(\varphi(\zeta)) \equiv O .
$$

By the very definition of $\Omega_{D, p}\left(\right.$ Equation (1.2) ) it follows $\Omega_{D, p}\left(F^{-1}(\zeta, w)\right)=\|w\|^{2}-$ $\operatorname{lm} \zeta$ for $(\zeta, w) \in \mathbb{C} \times \mathbb{C}^{n},(\zeta, w) \in \mathbb{H}^{n}$. Therefore

$$
\Omega_{D, p}(z)=\Omega_{D, p}\left(F^{-1}(F(z))\right)=\|\tilde{F}(z)\|^{2}-\operatorname{Im} F_{0}(z) .
$$


Thus, from (4.1) and (4.2) we have for $v \in \mathbb{C}^{n}$

$$
\operatorname{Hess}\left(\Omega_{D, p}\right)_{\varphi(\zeta)}(v, v)=2\left\|d \tilde{F}_{\varphi(\zeta)}(v)\right\|^{2}-\operatorname{Hess}\left(\operatorname{Im} F_{0}\right)_{\varphi(\zeta)}(v, v)
$$

where, for a real function $f$, Hess $(f)_{x}$ denotes the real Hessian of $f$ at $x$.

Now, let $r \in \mathbb{D} \cap \mathbb{R}$ and let $\theta_{r} \in \operatorname{Aut}(\mathbb{D})$ be such that $\theta_{r}(0)=r$ and $\theta_{r}(1)=1$ (notice that necessarily $\theta_{r}^{\prime}(0) \in \mathbb{R}$ ). Let $\widehat{\varphi \circ \theta_{r}}: \mathbb{D} \rightarrow D$ be the dual map of $\varphi \circ \theta_{r}$. From the very definition, a direct computation shows that

$$
\widetilde{\varphi \circ \theta_{r}}(\zeta)=\frac{\widetilde{\varphi}\left(\theta_{r}(\zeta)\right)}{\theta_{r}^{\prime}(\zeta)}
$$

By [34, Lemma 4.1] (and since $\theta_{r}^{\prime}(0) \in \mathbb{R}$ ) it follows that if $\varphi(r) \in \partial E_{D}(p, R(r))$, then

$$
\begin{aligned}
T_{\varphi(r)}\left(\partial E_{D}(p, R(r))\right) & =\left\{v \in \mathbb{C}^{n}: \operatorname{Re}\left(\widetilde{\varphi \circ \theta_{r}}(0) \cdot v\right)=0\right\} \\
& =\left\{v \in \mathbb{C}^{n}: \operatorname{Re}(\widetilde{\varphi}(r) \cdot v)=0\right\} .
\end{aligned}
$$

On the other hand, by (4.2) and (4.1) it follows that

$$
T_{\varphi(r)}\left(\partial E_{D}(p, R(r))\right)=\operatorname{ker} d\left(\Omega_{D, p}\right)_{\varphi(r)}=\operatorname{ker} d\left(\operatorname{Im} F_{0}\right)_{\varphi(r)} .
$$

Thus, since they have the same kernel, the two (real) forms $v \mapsto \operatorname{Re}(\widetilde{\varphi}(r) \cdot v)$ and $v \mapsto \operatorname{Im} d\left(F_{0}\right)_{\varphi(r)}(v)$ are multiples of each other. Since $\operatorname{Re}\left(\widetilde{\varphi}(r) \cdot \varphi^{\prime}(r)\right)=1$ by (1.1), and by (4.1)

$$
\operatorname{Im} d\left(F_{0}\right)_{\varphi(r)}\left(\varphi^{\prime}(r)\right)=\left.\operatorname{Im} \frac{d}{d \xi} F_{0}(\varphi(\xi))\right|_{\xi=r}=\left.\operatorname{Re} \frac{d}{d \xi} \frac{1+\xi}{1-\xi}\right|_{\xi=r}=\frac{2}{(1-r)^{2}}
$$

it follows that for all $v \in \mathbb{C}^{n}$

$$
d\left(\operatorname{Im} F_{0}\right)_{\varphi(r)}(v)=\frac{2 \operatorname{Re}(\tilde{\varphi}(r) \cdot v)}{(1-r)^{2}} .
$$

Now, let $R>0$ be such that $q \in \partial E_{D}(p, R)$ and assume that $v \in T_{q} \partial E_{D}(p, R)$ verifies $\operatorname{Hess}\left(\Omega_{D, p}\right)_{q}(v, v)=0$. We want to show that $v=0$.

Write $(\lambda, U)=\left(d\left(F_{0}\right)_{q}(v), d(\tilde{F})_{q}(v)\right)$. Since the map $\Phi_{p}$ transforms boundaries of horospheres onto boundaries of horospheres, it follows that the vector $(\lambda, U)$ is tangent to the boundary of the horosphere $\left\{(\zeta, w) \in \mathbb{C} \times \mathbb{C}^{n-1}: \operatorname{Im} \zeta-\|w\|^{2}>1\right\}$ whose closure contains $(i, O) \in \mathbb{H}^{n}$. Thus $\lambda \in \mathbb{R}$.

Let us now consider the smooth one-parameter family of complex geodesics $g_{t}$ : $\mathbb{D} \rightarrow \mathbb{H}^{n}$ depending smoothly on $t$ given by

$$
g_{t}(\zeta):=\left(i \frac{1+\zeta}{1-\zeta}+t \lambda+i t^{2}\|U\|^{2}, t U\right)
$$

and we denote

$$
\dot{g}(\zeta):=\left.\frac{\partial g_{t}(\zeta)}{\partial t}\right|_{t=0}, \quad \ddot{g}(\zeta):=\left.\frac{\partial^{2} g_{t}(\zeta)}{\partial^{2} t}\right|_{t=0}
$$

Notice that $\dot{g}(\zeta)=(\lambda, U)$ and $\ddot{g}(\zeta)=\left(2 i\|U\|^{2}, O\right)$ are independent of $\zeta$. Let $f_{t}:=F^{-1}\left(g_{t}\right)$. By construction $f_{t}: \mathbb{D} \rightarrow D$ is a smooth one-parameter family of complex geodesics, $f_{t}(1)=p$ and $f_{0}(0)=q$. Therefore $f_{0}=\varphi$. Again, denoting by $\dot{f}(\zeta), \ddot{f}(\zeta)$ the derivative of $f_{t}(\zeta)$ with respect to $t$ at $t=0$, it follows that $\dot{f}(1)=0$ and $\ddot{f}(1)=0$ because $f_{t}(1)=p$ for all $t$ (see Corollary 2.4).

Let us denote by $J(\zeta)$ the Jacobi vector field $J(\zeta):=\dot{f}(\zeta)$ along $\varphi$. We can write $J(\zeta)=\lambda(\zeta) \varphi^{\prime}(\zeta)+J^{\perp}(\zeta)$ for some holomorphic function $\lambda$ and vector field 
$J^{\perp}$ such that $\widetilde{\varphi}(\zeta) \cdot J^{\perp}(\zeta) \equiv 0$ and $\lambda(\zeta)=\alpha+i \beta \zeta-\bar{\alpha} \zeta^{2}$ for some $\alpha \in \mathbb{C}$ and $\beta \in \mathbb{R}$ (see [34, Theorem 3.1.c]).

Since $\dot{f}(1)=0$ then $J(1)=0$. Since the map $\Phi_{p}$ transforms boundaries of horospheres onto boundaries of horospheres, it follows that $J(0) \in T_{\varphi(0)}\left(\partial E_{D}(p, R)\right)$. In other words, by (4.4), $\operatorname{Re}(\widetilde{\varphi}(0) \cdot J(0))=0$, which implies that $\operatorname{Re} \alpha=0$ for $\widetilde{\varphi}(0) \cdot \varphi^{\prime}(0)=1$. Therefore $J(\zeta)=i \gamma(1-\zeta)^{2} \varphi^{\prime}(\zeta)+J^{\perp}(\zeta)$ with $\gamma \in \mathbb{R}$ and $J^{\perp}(1)=0$. This implies that $\operatorname{Re}(\widetilde{\varphi}(r) \cdot J(r))=0$ for $r \in(-1,1)$. Hence by (4.4) it follows that $J(r) \in T_{\varphi(r)}\left(\partial E_{D}(p, R(r))\right)$, where $R(r)>0$ is such that $\varphi(r) \in \partial E_{D}(p, R(r))$. Since boundaries of horospheres are convex, we have

$$
\operatorname{Hess}\left(\Omega_{D, p}\right)_{\varphi(r)}(J(r), J(r)) \geq 0, \quad r \in(-1,1) .
$$

Now differentiating with respect to $t$ the identity $F \circ f_{t}=g_{t}$ and setting $t=0$ we obtain

$$
\begin{aligned}
& d \tilde{F}_{\varphi(\zeta)}(J(\zeta))=U, \\
& \operatorname{Hess}\left(\operatorname{Im} F_{0}\right)_{\varphi(\zeta)}(J(\zeta), J(\zeta))+d\left(\operatorname{Im} F_{0}\right)_{\varphi(\zeta)}(\ddot{f}(\zeta))=\operatorname{Im} \ddot{g}(\zeta)=2\|U\|^{2} .
\end{aligned}
$$

Putting together (4.3), (4.5) and (4.7), we obtain for $r \in(-1,1)$

$$
\operatorname{Hess}\left(\Omega_{D, p}\right)_{\varphi(r)}(J(r), J(r))=\frac{\operatorname{Re} \widetilde{\varphi}(r) \cdot \ddot{f}(r)}{(1-r)^{2}} .
$$

Our next aim is to compute $\widetilde{\varphi}(r) \cdot \ddot{f}(r)$. In order to do this, we choose a suitable defining function: according to Pang [26, Proposition 2.36] there exists a $C^{\infty}$ defining function $\rho$ for $D$ near $\varphi(\bar{D})$ such that for all $\theta \in \mathbb{R}$ it follows that $\widetilde{\varphi}\left(e^{i \theta}\right)=e^{i \theta} \partial \rho_{\varphi\left(e^{i \theta}\right)}$. For all $t$ and for all $\theta \in \mathbb{R}$ it follows that $\rho\left(f_{t}\left(e^{i \theta}\right)\right) \equiv 0$. Thus differentiating such an identity (as we can, by Corollary 2.4) with respect to $t$ at $t=0$ we obtain $2 \operatorname{Re}\left(\partial \rho \cdot \ddot{f}\left(e^{i \theta}\right)\right)+\operatorname{Hess}(\rho)_{e^{i \theta}}\left(J\left(e^{i \theta}\right), J\left(e^{i \theta}\right)\right) \equiv 0$; namely,

$$
\operatorname{Re}(\bar{\zeta} \widetilde{\varphi}(\zeta) \cdot \ddot{f}(\zeta))=-\frac{1}{2} \operatorname{Hess}(\rho)_{\zeta}(J(\zeta), J(\zeta)), \quad|\zeta|=1 .
$$

Now, the function $\zeta \mapsto \widetilde{\varphi}(\zeta) \cdot \ddot{f}(\zeta)$ is holomorphic in $\mathbb{D}$. Write $\widetilde{\varphi}(\zeta) \cdot \ddot{f}(\zeta)=$ $A+\zeta B+\zeta^{2} C(\zeta)$ for some $A, B \in \mathbb{C}$ and some holomorphic function $C$. Then

$$
\operatorname{Re}(\bar{\zeta} \widetilde{\varphi}(\zeta) \cdot \ddot{f}(\zeta))=\operatorname{Re}(\bar{A} \zeta+B+\zeta C(\zeta)), \quad|\zeta|=1 .
$$

Let $T_{1}$ denote the Hilbert transform which associates to any harmonic function $u$ in $\mathbb{D}$, Hölder continuous on $\partial \mathbb{D}$, its harmonic conjugated $T_{1}(u)$, still Hölder continuous on $\partial \mathbb{D}$, normalized so that $T_{1}(u)(1)=0$. Let $h$ denote the holomorphic function in $\mathbb{D}$ whose trace on $\partial \mathbb{D}$ is $-1 / 2\left(\right.$ id $\left.+i T_{1}\right)(\operatorname{Hess}(\rho)(J, J))$. Notice that $\operatorname{Re} h \leq 0$ on $\partial \mathbb{D}$ since $\partial D$ is convex. Moreover, since $J(1)=0$ and by the normalization chosen for $T_{1}$, it follows that $h(1)=0$.

By (4.9) and (4.10) we obtain that $h(\zeta)=\bar{A} \zeta+B+\zeta C(\zeta)+i \alpha$ for some $\alpha \in \mathbb{R}$. Hence $\widetilde{\varphi}(\zeta) \cdot \ddot{f}(\zeta)-\zeta h(\zeta)=-\bar{A} \zeta^{2}-i \alpha \zeta+A$ and, since $h(1)=\ddot{f}(1)=0$, we get $-\bar{A}-i \alpha+A=0$. Writing $A=a+i b$ with $a, b \in \mathbb{R}$, we obtain

$$
\widetilde{\varphi}(\zeta) \cdot \ddot{f}(\zeta)=\zeta h(\zeta)+a\left(1-\zeta^{2}\right)+i b(1-\zeta)^{2} .
$$

Substituting this expression in (4.8) for $\zeta=r \in(-1,1)$, we find

$$
\operatorname{Hess}\left(\Omega_{D, p}\right)_{\varphi(r)}(J(r), J(r))=a \frac{1+r}{1-r}+\frac{r \operatorname{Re}(h(r))}{(1-r)^{2}} .
$$

By construction, $a=\operatorname{Hess}\left(\Omega_{D, p}\right)_{\varphi(0)}(J(0), J(0))=\operatorname{Hess}\left(\Omega_{D, p}\right)_{q}(v, v)=0$. By (4.6) and (4.11) it follows then that $\operatorname{Re}(h(r)) \geq 0$ for $r \in(0,1)$. However $\operatorname{Re} h(\zeta)$ 
is harmonic on $\mathbb{D}$ and non-positive on $\partial \mathbb{D}$, and thus by the maximum principle $\operatorname{Re} h(\zeta) \equiv 0$. Thus

$$
\operatorname{Hess}(\rho)_{\zeta}(J(\zeta), J(\zeta))=0, \quad \zeta \in \partial \mathbb{D}
$$

and, since $\partial D$ is strongly convex, it follows that $J=0$ on $\partial \mathbb{D}$ and thus $J \equiv 0$ on $\mathbb{D}$, proving that $v=0$.

\section{EXTREMALity}

Let $D \subset \mathbb{C}^{n}$ be a bounded strongly convex domain with smooth boundary. We let $\Gamma_{p}$ be the set of all $C^{\infty}$ curves $\gamma:[0,1] \rightarrow D \cup\{p\}$ such that $\gamma(1)=p$ and $\gamma^{\prime}(1) \notin T_{p} \partial D$ (notice that, if $\nu_{p}$ is the unit outward normal to $\partial D$ at $p$, then $\gamma^{\prime}(1) \notin T_{p} \partial D$ if and only if $\left.\operatorname{Re}\left\langle\gamma^{\prime}(1), \nu_{p}\right\rangle>0\right)$.

Theorem 5.1. Let $D \subset \mathbb{C}^{n}$ be a bounded strongly convex domain with smooth boundary and let $p \in \partial D$. Let $\nu_{p}$ be the unit outward normal to $\partial D$ at $p$. Consider the following family $\mathcal{S}_{p}(D)$ :

$$
\left\{\begin{array}{l}
u \in \operatorname{Psh}(D), \\
\limsup _{z \rightarrow x} u(z) \leq 0 \quad \text { for all } x \in \partial D \backslash\{p\}, \\
\liminf _{t \rightarrow 1}|u(\gamma(t))(1-t)| \geq 2 \operatorname{Re}\left(\left\langle\gamma^{\prime}(1), \nu_{p}\right\rangle^{-1}\right) \quad \text { for all } \gamma \in \Gamma_{p} .
\end{array}\right.
$$

Then $\Omega_{D, p} \in \mathcal{S}_{p}(D)$ (where $\Omega_{D, p}$ is the function defined in Theorem 0.1) and $u \leq \Omega_{D, p}$ for all $u \in \mathcal{S}_{p}(D)$.

To prove the theorem we need some preliminary results. Let subh $(\mathbb{D})$ denote the real cone of subharmonic functions in the unit disc $\mathbb{D}$.

Lemma 5.2 (Phragmen-Lindelöf). Let $c>0$. Consider the following family $\mathcal{S}_{c}(\mathbb{D})$ in the unit disc:

$$
\left\{\begin{array}{l}
u \in \operatorname{subh}(\mathbb{D}), \\
u<0 \quad \text { in } \mathbb{D} \\
\liminf _{\mathbb{R} \ni r \rightarrow 1^{-}}|u(r)(1-r)| \geq 2 c .
\end{array}\right.
$$

Then $-c P(\zeta) \in \mathcal{S}_{c}(\mathbb{D})$, and for all $u \in \mathcal{S}_{c}(\mathbb{D})$ it follows that $u \leq-c P(\zeta)$.

For the sake of completeness we give a short proof of Lemma [5.2, based on some notes of Professor P. Poggi-Corradini. We thank him for letting us use his notes.

Proof. It is clear that $-c P(\zeta) \in \mathcal{S}_{c}(\mathbb{D})$. We have to show that $-c P$ is the maximal element of the family.

First of all, let $C(\zeta)=(1+\zeta) \cdot(1-\zeta)^{-1}$ be the Cayley transformation from $\mathbb{D}$ to $\mathbb{H}=\{w \in \mathbb{C}: \operatorname{Re} w>0\}$. Then we consider the family $C^{*}\left(\mathcal{S}_{c}(\mathbb{D})\right)=\{\tilde{u}: \tilde{u}=$ $u \circ C^{-1}$ for some $\left.u \in \mathcal{S}_{c}(\mathbb{D})\right\}$. Then, if $\tilde{u} \in C^{*}\left(\mathcal{S}_{c}(\mathbb{D})\right)$ it follows that $\tilde{u} \in \operatorname{subh}(\mathbb{H})$, $\tilde{u}<0$ in $\mathbb{H}$ and

$$
\limsup _{\mathbb{R} \ni R \rightarrow+\infty} \frac{\tilde{u}(R)}{R} \leq-c
$$

Notice that $P \circ C^{-1}(w)=\operatorname{Re} w$ is the Poisson kernel in $\mathbb{H}$. Let $\tilde{u}=u \circ C^{-1} \in$ $C^{*}\left(\mathcal{S}_{c}(\mathbb{D})\right)$ and let $L=\lim \sup _{\mathbb{R} \ni R \rightarrow+\infty} \tilde{u}(R) / R \leq-c$. We are going to show that $\tilde{u} \leq L \operatorname{Re} w$, from which it follows that $u \leq-c P$. 
Let $\epsilon>0$ be such that $\epsilon<-L$. Let $v(w)=\tilde{u}(w)-(L+\epsilon) \operatorname{Re} w$. Now, $v \in \operatorname{subh}(\mathbb{H})$, $\lim \sup _{w \rightarrow i y} v(w) \leq 0$ for all $y \in \mathbb{R}$ and

$$
\limsup _{\mathbb{R} \ni R \rightarrow+\infty} v(R)=\limsup _{\mathbb{R} \ni R \rightarrow+\infty} R\left(\frac{\tilde{u}(R)}{R}-(L+\epsilon)\right) \leq 0 .
$$

Therefore there exists $\delta>0$ such that $v(R) \leq 1$ for $R \leq \delta$ and $R \geq \frac{1}{\delta}$. Moreover, since $v$ is semicontinuous, there exists $K>0$ such that $v(R) \leq K$ for $\delta<R<\frac{1}{\delta}$. We now consider $V(w)=v(\sqrt{i w})-K$. Again, $V \in \operatorname{subh}(\mathbb{H})$ and $\lim \sup _{w \rightarrow i y} V(w) \leq 0$ for all $y \in \mathbb{R}$. Moreover,

$$
\begin{aligned}
\sup _{-\pi / 2<\theta<\pi / 2} V\left(r e^{i \theta}\right) & =\sup _{0<\theta<\pi / 2} v\left(\sqrt{r} e^{i \theta}\right)-K \\
& =\sup _{0<\theta<\pi / 2}\left[\tilde{u}\left(\sqrt{r} e^{i \theta}\right)-(L+\epsilon) \sqrt{r} \cos \theta-K\right] \\
& \leq \sup _{0<\theta<\pi / 2}(-(L+\epsilon) \sqrt{r} \cos \theta-K) \\
& =-(L+\epsilon) \sqrt{r}-K .
\end{aligned}
$$

By the classical estimates on sub-linear growth of subharmonic functions (see, e.g., [30), it follows that $V(w) \leq 0$ for all $w \in \mathbb{H}$, and therefore $v \leq K$ in the first quadrant. A similar argument shows that $v \leq K$ on the fourth quadrant, and as before, $v \leq 0$ on $\mathbb{H}$ which implies $\tilde{u}(w) \leq(L+\epsilon) \operatorname{Re} w$ for $w \in \mathbb{H}$. By the arbitrariness of $\epsilon$ we have the statement.

Proof of Theorem 5.1. Up to rigid movements, we can suppose that $\nu_{p}=e_{1}$.

First of all, notice that the function identically 0 does not belong to $\mathcal{S}_{p}(D)$ because of the estimates at $p$.

We claim that if $u \in \mathcal{S}_{p}(D)$, then $u<0$ in $D$. Indeed, let $\varphi: \mathbb{D} \rightarrow D$ be a complex geodesic not containing $p$ in its closure (in fact, any attached analytic disc not containing $p$ would be enough). Then $\tilde{u}=u \circ \varphi: \mathbb{D} \rightarrow \mathbb{R}$ is subharmonic and $\lim \sup _{\zeta \rightarrow x} \tilde{u}(\zeta) \leq 0$ for all $x \in \partial \mathbb{D}$. Thus by the maximum principle for subharmonic functions, $\tilde{u} \leq 0$ in $\mathbb{D}$ and hence $u \leq 0$ in $D$, as $\varphi$ was an arbitrary complex geodesic. Again, the maximum principle for plurisubharmonic functions implies that $u<0$ in $D$ or $u \equiv 0$, and the latter cannot be the case. Thus $u<0$ in $D$ as wanted.

Now, let $v \in L_{p}$ and let $\varphi_{v}: \mathbb{D} \rightarrow D$ be a complex geodesic parameterized in the Chang-Hu-Lee normal parametrization. Let $\widetilde{\rho}_{v}: D \rightarrow \mathbb{D}$ be its left inverse. We show that the function $u_{v}: D \rightarrow \mathbb{R}^{-}$defined by

$$
u_{v}(z)=-\frac{P\left(\widetilde{\rho}_{v}(z)\right)}{v_{1}^{2}}
$$

belongs to $\mathcal{S}_{p}(D)$. It is clear that $u_{v} \in \operatorname{Psh}(D), \limsup _{z \rightarrow x} u_{v}(z) \leq 0$ for all $x \in \partial D \backslash\{p\}$ since $\tilde{\rho}_{v}\left(\bar{D} \backslash \varphi_{v}(\partial \mathbb{D})\right) \subset \mathbb{D}$. We claim that for all smooth curves $\gamma:[0,1] \rightarrow D \cup\{p\}$ such that $\gamma(1)=p$ and $\left\langle\gamma^{\prime}(1), e_{1}\right\rangle \neq 0$ (that is, $\gamma^{\prime}(1)$ is not complex tangential to $\partial D$ ), it follows

$$
\lim _{t \rightarrow 1}\left|u_{v}(\gamma(t))(1-t)\right|=\frac{2 \operatorname{Re}\left\langle\gamma^{\prime}(1), e_{1}\right\rangle}{\left|\left\langle\gamma^{\prime}(1), e_{1}\right\rangle\right|^{2}} .
$$

Indeed,

$$
\left|u_{v}(\gamma(t))(1-t)\right|=\frac{1}{v_{1}^{2}} \frac{1-\left|\widetilde{\rho}_{v}(\gamma(t))\right|^{2}}{1-t} \frac{(1-t)^{2}}{\left|1-\widetilde{\rho}_{v}(\gamma(t))\right|^{2}}
$$


Now

$$
\lim _{t \rightarrow 1} \frac{1-\widetilde{\rho}_{v}(\gamma(t))}{1-t}=\left.\frac{d}{d t}\left(\widetilde{\rho}_{v}(\gamma(t))\right)\right|_{t=1}=d\left(\widetilde{\rho}_{v}\right)_{p}\left(\gamma^{\prime}(1)\right)=\frac{\left\langle\gamma^{\prime}(1), e_{1}\right\rangle}{\left\langle\varphi_{v}^{\prime}(1), e_{1}\right\rangle}
$$

where the last equality follows from (3.7) and because $\varphi_{v}^{\prime}(1)=v_{1} v$. Moreover

$$
\begin{aligned}
\lim _{t \rightarrow 1} \frac{1-\left|\widetilde{\rho}_{v}(\gamma(t))\right|^{2}}{1-t} & =\left.\frac{d}{d t}\left(\left|\widetilde{\rho}_{v}(\gamma(t))\right|^{2}\right)\right|_{t=1} \\
& =\left.\widetilde{\rho}_{v}(\gamma(1)) \frac{d}{d t}\left(\overline{\widetilde{\rho}_{v}(\gamma(t))}\right)\right|_{t=1}+\left.\overline{\widetilde{\rho}_{v}(\gamma(1))} \frac{d}{d t}\left(\widetilde{\rho}_{v}(\gamma(t))\right)\right|_{t=1} \\
& =\left.2 \operatorname{Re} \frac{d}{d t}\left(\widetilde{\rho}_{v}(\gamma(t))\right)\right|_{t=1}=2 \operatorname{Re} \frac{\left\langle\gamma^{\prime}(1), e_{1}\right\rangle}{\left\langle\varphi_{v}^{\prime}(1), e_{1}\right\rangle}
\end{aligned}
$$

Therefore

$$
\lim _{t \rightarrow 1}\left|u_{v}(\gamma(t))(1-t)\right|=\frac{2}{v_{1}^{2}} \operatorname{Re} \frac{\left\langle\gamma^{\prime}(1), e_{1}\right\rangle}{\left\langle\varphi_{v}^{\prime}(1), e_{1}\right\rangle} \cdot \frac{\left|\left\langle\varphi_{v}^{\prime}(1), e_{1}\right\rangle\right|^{2}}{\left|\left\langle\gamma^{\prime}(1), e_{1}\right\rangle\right|^{2}} .
$$

Taking into account that $\left\langle\varphi_{v}^{\prime}(1), e_{1}\right\rangle=v_{1}^{2}$, we have the claim. In particular equation (5.4) holds if $\gamma \in \Gamma_{p}$, showing that $u_{v}$ belongs to $\mathcal{S}_{p}(D)$.

Notice that $\Omega_{D, p}\left(\varphi_{v}(\zeta)\right)=u_{v}\left(\varphi_{v}(\zeta)\right)$ for all $\zeta \in \mathbb{D}$. Moreover, if $u \in \mathcal{S}_{p}(\mathbb{D})$, then $\tilde{u}: \zeta \mapsto u\left(\varphi_{v}(\zeta)\right)$ is in the family $\mathcal{S}_{1 / v_{1}^{2}}(\mathbb{D})$ given by (5.2) (with $c=1 / v_{1}^{2}$ ). Indeed, $\tilde{u} \in \operatorname{subh}(\mathbb{D}), u<0$, in $\mathbb{D}$ and

$$
\liminf _{\mathbb{R} \ni r \rightarrow 1}|\tilde{u}(r)|(1-r) \geq \frac{2 \operatorname{Re}\left\langle\varphi_{v}^{\prime}(1), e_{1}\right\rangle}{\left|\left\langle\varphi_{v}^{\prime}(1), e_{1}\right\rangle\right|^{2}}=\frac{2}{\left\langle\varphi_{v}^{\prime}(1), e_{1}\right\rangle}=\frac{2}{v_{1}^{2}},
$$

since $\varphi_{v}^{\prime}(1)=v_{1}^{2} e_{1}+e_{1}^{\perp}$. Thus, by Lemma 5.2 it follows that for all $\zeta \in \mathbb{D}$

$$
u\left(\varphi_{v}(\zeta)\right)=\tilde{u}(\zeta) \leq \frac{-1}{v_{1}^{2}} P(\zeta)=\frac{-1}{v_{1}^{2}} u_{v}\left(\varphi_{v}(\zeta)\right)=\Omega_{D, p}\left(\varphi_{v}(\zeta)\right) .
$$

Thus for all $u \in \mathcal{S}_{p}(D)$ we have $u \leq \Omega_{D, p}$. It remains only to show that $\Omega_{D, p} \in$ $\mathcal{S}_{p}(D)$. To this aim, we let $\varphi_{v_{t}}: \mathbb{D} \rightarrow D$ be the complex geodesic in Chang-HuLee normal parametrization such that $\gamma(t) \in \varphi_{v_{t}}(\mathbb{D})$. Moreover, we denote it by $v_{t}=\varphi_{v_{t}}^{\prime}(1) \in L_{p}$. Thus

$$
\Omega_{D, p}(\gamma(t))=u_{v_{t}}(\gamma(t))=\frac{-1}{\left\langle v_{t}, e_{1}\right\rangle^{2}} P\left(\widetilde{\rho}_{v_{t}}(\gamma(t))\right)
$$

Hence

$$
\left|\Omega_{D, p}(\gamma(t))\right|(1-t)=\frac{1}{\left\langle v_{t}, e_{1}\right\rangle^{2}} \frac{1-\left|\widetilde{\rho}_{v_{t}}(\gamma(t))\right|^{2}}{1-t} \frac{(1-t)^{2}}{\left|1-\widetilde{\rho}_{v_{t}}(\gamma(t))\right|^{2}}
$$

Fix $v=v_{t}$. By the mean value theorem it follows that

$$
\frac{1-\operatorname{Re} \widetilde{\rho}_{v}(\gamma(t))}{1-t}=\left.\frac{d}{d t} \operatorname{Re} \widetilde{\rho}_{v}(\gamma(t))\right|_{t=s}=\operatorname{Re} d\left(\widetilde{\rho}_{v}\right)_{\gamma(s)}\left(\gamma^{\prime}(s)\right),
$$

for some $t<s<1$, and similarly for the imaginary part and for the modulus $\left|\widetilde{\rho}_{v_{t}}(\gamma(t))\right|^{2}$. Notice that $s$ depends on $v$, but clearly $s \rightarrow 1$ as $t \rightarrow 1$.

Now let $\left\{v_{t_{k}}\right\}$ be a converging subsequence of $\left\{v_{t}\right\}$. By Lemma 3.4 if $v_{t_{k}} \rightarrow v_{0}$, then $v_{0} \in L_{p}$ (and in particular $\left\langle v_{t_{k}}, e_{1}\right\rangle^{2} \rightarrow\left\langle v_{0}, e_{1}\right\rangle^{2}>0$ ). Therefore, by Lemma 3.5 we have

$$
\lim _{t \rightarrow 1} d\left(\widetilde{\rho}_{v_{t}}\right)_{\gamma(s)}\left(\gamma^{\prime}(s)\right)=d\left(\widetilde{\rho}_{v_{0}}\right)_{p}\left(\gamma^{\prime}(1)\right)
$$


Thus by (5.5) and (3.7) it follows that

$$
\lim _{t_{k} \rightarrow 1}\left|\Omega_{D, p}\left(\gamma\left(t_{k}\right)\right)\right|\left(1-t_{k}\right)=\frac{1}{\left\langle v_{0}, e_{1}\right\rangle^{2}} \frac{2 \operatorname{Re} d\left(\widetilde{\rho}_{v_{0}}\right)_{p}\left(\gamma^{\prime}(1)\right)}{\left|d\left(\widetilde{\rho}_{v_{0}}\right)_{p}\left(\gamma^{\prime}(1)\right)\right|^{2}}=\frac{2 \operatorname{Re}\left\langle\gamma^{\prime}(1), e_{1}\right\rangle}{\left|\left\langle\gamma^{\prime}(1), e_{1}\right\rangle\right|^{2}} .
$$

Since this holds for any converging subsequence of $\left\{v_{t}\right\}$, we have that

$$
\lim _{t \rightarrow 1}\left|\Omega_{D, p}(\gamma(t))\right|(1-t)=\frac{2 \operatorname{Re}\left\langle\gamma^{\prime}(1), e_{1}\right\rangle}{\left|\left\langle\gamma^{\prime}(1), e_{1}\right\rangle\right|^{2}} .
$$

Corollary 5.3. Let $\Omega_{D, p}$ be the function given by Theorem 0.1 . Then for all smooth curves $\gamma:[0,1] \rightarrow D \cup\{p\}$ such that $\gamma(1)=p$ and $\gamma^{\prime}(1) \notin T_{p}^{\mathbb{C}} \partial D$, it follows that

$$
\lim _{t \rightarrow 1}\left|\Omega_{D, p}(\gamma(t))\right|(1-t)=\operatorname{Re} \frac{2}{\left\langle\gamma^{\prime}(1), \nu_{p}\right\rangle} .
$$

Proof. If $\gamma^{\prime}(1) \notin T_{p} \partial D$, then the claim follows from the proof of Theorem 5.1 . In case $\gamma^{\prime}(1) \in T_{p} \partial D \backslash T_{p}^{\mathbb{C}} \partial D$ - that is, $\operatorname{Re}\left\langle\gamma^{\prime}(1), \nu_{p}\right\rangle=0$ but $\left\langle\gamma^{\prime}(1), \nu_{p}\right\rangle \neq 0$-let $v \in L_{p}$ and let $u_{v}$ be given by (5.3). By Theorem 5.1 it follows that for all $z \in D$

$$
0 \leq\left|\Omega_{D, p}(z)\right| \leq\left|u_{v}(z)\right| .
$$

By (5.4) it follows that $\left|u_{v}(\gamma(t))\right|(1-t) \rightarrow 0$ and then $\left|\Omega_{D, p}(\gamma(t))\right|(1-t) \rightarrow 0$, proving the statement.

\section{Green's versus Poisson's Pluricomplex functions}

Let $D$ be a bounded strongly convex domain in $\mathbb{C}^{n}$ with smooth boundary and let $z_{0} \in D$. Consider the problem in (0.1). In his outstanding work [21, 24, Lempert proved that there exists a unique solution $L_{D, z_{0}}$, given by $L_{D, z_{0}}=\log \left\|\Phi_{z_{0}}\right\|$, where $\Phi_{z_{0}}: D \rightarrow \mathbb{B}^{n}$ is the Lempert spherical representation with center $z_{0}$ introduced in Section 1. Rephrasing the very definition of $\Phi_{z_{0}}$, it follows that for $z \in D$

$$
L_{D, z_{0}}(z)=\log \left(\tanh k_{D}\left(z_{0}, z\right)\right) .
$$

We have the following relations between the pluricomplex Green function $L_{D, z_{0}}$ and the pluricomplex Poisson kernel $\Omega_{D, p}$ solution of problem (0.2) which generalizes the corresponding relation in $\mathbb{D}$ between the classical Green function and the classical Poisson kernel (see for instance [20, Proposition 2.2.2]):

Theorem 6.1. Let $D$ be a bounded strongly convex domain in $\mathbb{C}^{n}$ with smooth boundary. Let $z_{0} \in D$ and $p \in \partial D$. Let $\nu_{p}$ be the outer normal of $\partial D$ at $p$. Then

$$
\Omega_{D, p}\left(z_{0}\right)=-\frac{\partial L_{D, z_{0}}}{\partial \nu_{p}}(p) .
$$

Proof. Let $K_{z_{0}}:=\exp \left(L_{D, z_{0}}\right)$. Let $\varphi: \mathbb{D} \rightarrow D$ be a complex geodesic such that $\varphi(0)=z_{0}$ and $\varphi(1)=p$. Since $\frac{\partial K_{z}}{\partial \nu_{p}}(p)>0$ for all $z \in D$, by [1, Theorem 2.6.47] (see also 4]) it follows that

$$
\lim _{\mathbb{R} \ni t \rightarrow 1}\left[k_{D}(z, \varphi(t))-k_{D}\left(z_{0}, \varphi(t)\right)\right]=\frac{1}{2}\left[\log \frac{\partial K_{z_{0}}}{\partial \nu_{p}}(p)-\log \frac{\partial K_{z}}{\partial \nu_{p}}(p)\right] .
$$

On the other hand by [11, Proposition 7.1]

$$
\lim _{\mathbb{R} \ni t \rightarrow 1}\left[k_{D}(z, \varphi(t))-k_{D}\left(z_{0}, \varphi(t)\right)\right]=\frac{1}{2}\left[\log \left|\Omega_{D, p}\left(z_{0}\right)\right|-\log \left|\Omega_{D, p}(z)\right|\right],
$$


which implies that there exists $C>0$ such that for all $z \in D$

$$
\Omega_{D, p}(z)=-C \frac{\partial K_{z}}{\partial \nu_{p}}(p) .
$$

We want to show that $C=1$. Let $\varphi: \mathbb{D} \rightarrow D$ be the unique complex geodesic in Chang-Hu-Lee normal parametrization such that $\varphi(1)=p$ and $\varphi^{\prime}(1)=\nu_{p}$. By the very definition $\Omega_{D, p}(\varphi(\zeta))=-P(\zeta)$, where $P$ is the Poisson kernel of $\mathbb{D}$ and $K_{\varphi(0)}(\varphi(\zeta))=|\zeta|$ for all $\zeta \in \mathbb{D}$. Since

$$
\frac{\partial K_{\varphi(0)}}{\partial \nu_{p}}(p)=\left.\frac{d}{d r}\left(K_{\varphi(0)} \circ \varphi(r)\right)\right|_{r=0}=\frac{d}{d r} r=1
$$

and $P(0)=1$, it follows that $C=1$, as wanted. Finally, since $\frac{\partial K_{z}}{\partial \nu_{p}}(p)=K_{z}(p) \frac{\partial L_{D, z}}{\partial \nu_{p}}$ and $K(p)=1$ for $p \in \partial D$, we get (6.2) .

\section{UNIQUENESS PROPERTIES}

In this section we study some analytical and geometrical properties which characterize the pluricomplex Poisson kernel introduced before.

Before we start, recall that, according to Bedford and Taylor [8] (see also [20. Section 3.5], the complex Monge-Ampère operator $\left(d d^{c}\right)^{n}$ (here $d^{c}=i(\bar{\partial}-\partial)$ ) can be defined for all $u \in \operatorname{Psh}(D) \cap L_{l o c}^{\infty}(D)$ for any bounded domain $D \subset \mathbb{C}^{n}$. Moreover, if $u \in \operatorname{Psh}(D) \cap L_{l o c}^{\infty}(D)$, then $\left(d d^{c} u\right)^{n}=(\partial \bar{\partial} u)^{n}=0$ if and only if $u$ is maximal in $D$; namely, for all relatively compact open subsets $E \subset D$ and all plurisubharmonic functions $v$ in $E$ such that $\lim _{\sup _{E \ni z \rightarrow x}} v(z) \leq u(x)$ for all $x \in \partial E$, it follows that $v \leq u$ in $E$.

Now we can state and prove the first uniqueness result, which is analogous in our setting of the uniqueness statement for the Monge-Ampère equation with one concentrated logarithmic singularity in the domain $D$ (see [24]).

Theorem 7.1. Let $D \subset \mathbb{C}^{n}$ be a bounded strongly convex domain with smooth boundary and let $p \in \partial D$. Let $u \in \operatorname{Psh}(D) \cap L_{l o c}^{\infty}(D)$ be such that $(\partial \bar{\partial} u)^{n}=0$, $\lim _{z \rightarrow x} u(z)=0$ for all $x \in \partial D \backslash\{p\}$ and

$$
\lim _{z \rightarrow p} \frac{u(z)}{\Omega_{D, p}(z)}=1 \text {. }
$$

Then $u \equiv \Omega_{D, p}$.

Proof. First of all we notice that (7.1) implies that $u$ belongs to the family (5.1) because for all $\gamma \in \Gamma_{p}$ (here $\Gamma_{p}$ is the set of curves defined in Section 5) it follows that

$$
\lim _{t \rightarrow 1} u(\gamma(t))(1-t)=\lim _{t \rightarrow 1} \frac{u(\gamma(t))}{\Omega_{D, p}(\gamma(t))} \Omega_{D, p}(\gamma(t))(1-t)=-\operatorname{Re} \frac{2}{\left\langle\gamma^{\prime}(1), \nu_{p}\right\rangle} .
$$

Therefore, by Theorem 5.1 it follows that $u(z) \leq \Omega_{D, p}(z)$ for all $z \in D$. Suppose that $u\left(z_{0}\right)<\Omega_{D, p}\left(z_{0}\right)$ for some $z_{0} \in D$. Then there exist $0<c<1$ and $\delta>0$ such that the set

$$
E_{\delta, c}:=\left\{z \in D: \Omega_{D, p}(z)>c u(z)+\delta\right\}
$$

is non-empty. Since $u$ is upper semi-continuous the set $E_{\delta, c}$ is open. If we prove that $E_{\delta, c}$ is relatively compact in $D$, since $(\partial \bar{\partial}(c u+\delta))^{n}=0$ and $\Omega_{D, p}(z) \leq c u(z)+\delta$ on $\partial E_{\delta, c}$, by maximality it follows that $\Omega_{D, p}(z) \leq c u(z)+\delta$ in $E_{\delta, c}$, contradicting the definition of $E_{\delta, c}$. 
Thus we are left to show that $E_{\delta, c}$ is relatively compact in $D$. First of all, since $u(x)=\Omega_{D, p}(x)=0$ for all $x \in \partial D \backslash\{p\}$, then $\overline{E_{\delta, c}} \subset D \cup\{p\}$. Seeking a contradiction, we assume that $p \in \overline{E_{\delta, c}}$. Thus there exists $\left\{z_{k}\right\} \subset E_{\delta, c}$ such that $z_{k} \rightarrow p$. Therefore for all $k \in \mathbb{N}$

$$
\Omega_{D, p}\left(z_{k}\right)-c u\left(z_{k}\right)-\delta>0 .
$$

Up to subsequences, we can assume that $\Omega_{D, p}\left(z_{k}\right) \rightarrow L$ for some $L \in[-\infty, 0]$. If $L<0$, then dividing (7.2) by $\Omega_{D, p}\left(z_{k}\right)<0$ and passing to the limit, taking into account (7.1), we would find $1-c \leq 0$, a contradiction since $c<1$. If $L=0$, (7.1) implies that $u\left(z_{k}\right) \rightarrow 0$ as $k \rightarrow \infty$, and therefore we reach a contradiction by passing to the limit for $k \rightarrow \infty$ in (7.2). Hence $p$ is not in the closure of $E_{\delta, c}$, which is thus relatively compact in $D$.

Remark 7.2. As pointed out in the Introduction, Theorem 7.1 is analogous of the uniqueness statement for problem (0.1), where uniqueness is established in the class of plurisubharmonic functions $u \in \operatorname{Psh}(D)$ such that $\lim _{z \rightarrow x} u(z)=0$ for all $x \in \partial D$ and $u(z)$ proceeds like the pluricomplex Green function $L_{D, z_{0}}$ for $z \rightarrow z_{0}$. Since for any convex domain the function $L_{D, z_{0}}$ proceeds like $\log \left\|z-z_{0}\right\|$ at $z_{0}$, then in the case of a inner singularity, there is a "universal" behavior. When the singularity is at $p \in \partial D$, it turns out that, thanks to Corollary [5.3, we know that the behavior of $\Omega_{D, p}$ along all non-tangential directions is independent of $D$, but we do not have any hint on the behavior of $\Omega_{D, p}$ along the tangential directions, which might depend on $D$ near $p$.

Next we characterize the pluricomplex Poisson kernel in terms of its associated Monge-Ampère foliation, with no hypotheses on the behavior near the boundary singularity.

Theorem 7.3. Let $D \subset \mathbb{C}^{n}$ be a bounded strongly convex domain with smooth boundary and let $p \in \partial D$. Let $u \in \operatorname{Psh}(D) \cap C^{2}(D)$ be such $\lim _{z \rightarrow x} u(z)=0$ for all $x \in \partial D \backslash\{p\}$. Then the restriction of $u$ to each complex geodesic whose closure contains $p$ is harmonic if and only if there exists $c \geq 0$ such that $u=c \Omega_{D, p}$.

Proof. One direction is obvious. Assume then that $u \in \operatorname{Psh}(D) \cap C^{2}(D)$ is harmonic on each complex geodesic whose closure contains $p$ and $\lim _{z \rightarrow x} u(z)=0$ for all $x \in \partial D \backslash\{p\}$. Arguing as in the proof of Theorem 5.1] we see that $u<0$ in $D$ or $u \equiv 0$. In the latter case $c=0$, and the theorem is proved. Thus we can assume that $u<0$ in $D$.

First of all, it is a well known result that if $v \geq 0$ is a harmonic function in $\mathbb{D}$ such that $\lim _{\zeta \rightarrow x} v(\zeta)=0$ for all $x \in \partial \mathbb{D} \backslash\{1\}$, then $v=c P$ for some $c \geq 0$ (here, as usual, $P$ denotes the Poisson kernel).

Therefore $u=\lambda \Omega_{D, p}$ for some $C^{2}$ function $\lambda: D \rightarrow(0, \infty)$ which is constant on each complex geodesic whose closure contains $p$. We need to show that $\lambda$ is constant.

To this aim, we argue as in the proof of Theorem 4.1 and retain the notation introduced there. Let $q \in D$. Up to post-composing with automorphisms of $\mathbb{B}^{n}$ and with the Cayley transform, we let $F: D \rightarrow \mathbb{H}^{n}$ be the diffeomorphism defined by means of the boundary spherical representation $\Phi_{p}$, such that $F(q)=(i, O)$. We let $U=u \circ F^{-1}$. Then $U$ is a $C^{2}$ negative function on $\mathbb{H}^{n}$, and by the very definition of $\Omega_{D, p}$ and 11, Theorem 7.3], it follows that $U(\xi, w)=\tilde{\lambda}(w)\left(\|w\|^{2}-\operatorname{Im} \xi\right)$. We are going to prove that $w=O$ is a critical point for $\tilde{\lambda}$; from this, since $F$ is a 
diffeomorphism from $D$ to $\mathbb{H}^{n}$, it will follow that $\lambda$ has a critical point at $q=$ $F^{-1}(i, O)$ and, by the arbitrariness of $q$, it will follow that all points of $D$ are critical for $\lambda$, which turns out to be constant.

Since $\tilde{\lambda}$ is a real function, it is enough to prove that the vector valued function $V:=\left(\frac{\partial \tilde{\lambda}}{\partial w_{1}}(0), \ldots, \frac{\partial \tilde{\lambda}}{\partial w_{n-1}}(0)\right)$ is zero. Let $\varphi: \mathbb{D} \rightarrow D$ be the complex geodesic such that $\varphi(0)=q$ and $\varphi(1)=p$. According to [26, Section 2.39] we can assume to be working with a system of holomorphic coordinates $\left(z_{1}, \ldots, z_{n}\right)$ in a neighborhood of $\varphi(\overline{\mathbb{D}})$ for which (among other conditions on the defining function of $D$ which we only use implicitly when referring to the paper 32 in the course of the proof) $\varphi(\zeta)=(\zeta, 0, \ldots, 0)$ for $\zeta \in \mathbb{D}$.

By construction it follows that if we write $G:=F^{-1}=\left(G_{1}, \ldots, G_{n}\right)$, then $G_{1}(\xi, O)=(\xi-i) /(\xi+i)$ and $G_{j}(\xi, O)=0$ for $j>1$ and $\operatorname{Im} \xi>0$.

Now let $t \mapsto w(t)$ be a smooth curve in $\mathbb{C}^{n-1}$ such that $w(0)=O$. Let $g_{t}(\zeta):=$ $\left(i(1+\zeta) /(1-\zeta)+i\|w(t)\|^{2}, w(t)\right)$ for $\zeta \in \mathbb{D}$ and $t$ close to 0 . By definition, $\left\{g_{t}\right\}$ is a family of complex geodesics in $\mathbb{H}^{n}$, and thus $\varphi_{t}:=G\left(g_{t}(\zeta)\right)$ is a smooth real one-parameter family $\left\{\varphi_{t}\right\}$ of complex geodesics in $D$ such that $\varphi_{0}(\zeta)=(\zeta, O)$. The associated Jacobi vector field $J(\zeta)=\frac{\partial \varphi_{t}}{\partial t}(\zeta)$ can be written in the form

$$
J(\zeta)=J_{1}(\zeta) \frac{\partial}{\partial z_{1}}+J^{\perp}(\zeta),
$$

where $J^{\perp}(\zeta)=\sum_{k=2}^{n} J_{k}(\zeta) \frac{\partial}{\partial z_{k}}$, and, since $\varphi_{t}(1)=p$ for all $t$, by Corollary 2.4 it follows that $J(1)=O$. Therefore, from [32, Section 3] it follows that there exist $a \in \mathbb{C}, X, Y \in \mathbb{C}^{n-1}$ (depending on $J$ ) and a unique continuous map $M: \overline{\mathbb{D}} \rightarrow$ GL $(2 n-2, \mathbb{C})$ holomorphic in $\mathbb{D}$ which depends only on $D$ and $\varphi$ with the following properties. If $M(\zeta)=\left(\begin{array}{ll}M_{1}(\zeta) & M_{2}(\zeta) \\ M_{3}(\zeta) & M_{4}(\zeta)\end{array}\right)$ where the $M_{j}$ 's are suitable $(n-1) \times(n-1)$ matrices with $M_{1}(1)=\frac{1}{2}$ Id, $M_{2}(1)=\frac{-i}{2}$ Id (and $M_{3}(1), M_{4}(1)$ satisfy suitable conditions that we do not need here), then

$$
\begin{aligned}
J_{1}(\zeta) & =(1-\zeta)(a+\bar{a} \zeta), \\
J^{\perp}(\zeta) & =i(1-\zeta)\left(M_{1}(\zeta) X+M_{2}(\zeta) Y\right) .
\end{aligned}
$$

By the very definition of $G$ and by (7.3), taking into account that $G$ maps complex tangent spaces to the boundary of horospheres in $\mathbb{H}^{n}$ to complex tangent spaces to the boundary of horospheres in $D$ (see the proof of Theorem 6.3 in [11]), it follows that for $\operatorname{Im} \xi>0$ and $\zeta \in \mathbb{D}$

$$
\begin{aligned}
& \frac{\partial G_{j}}{\partial \bar{\xi}}(\xi, 0)=0 \text { for } j=1, \ldots, n, \\
& \frac{\partial G_{1}}{\partial \xi}(\xi, 0)=\frac{\partial}{\partial \xi}(\xi-i)(\xi+i)^{-1}, \\
& \frac{\partial G_{j}}{\partial \xi}(\xi, 0)=0 \text { for } j=2, \ldots, n, \\
& \frac{\partial G_{1}}{\partial w_{j}}(\xi, 0)=\frac{\partial G_{1}}{\partial \bar{w}_{j}}(\xi, 0)=0 \text { for } j=1, \ldots, n-1, \\
& \frac{\partial G_{k}}{\partial w_{j}}\left(i \frac{1+\zeta}{1-\zeta}, 0\right)=i(1-\zeta)\left(M_{1}(\zeta) S_{j}+M_{2}(\zeta) T_{j}\right)_{k} \quad \text { for } j, k=2, \ldots, n, \\
& \frac{\partial G_{k}}{\partial \bar{w}_{j}}\left(i \frac{1+\zeta}{1-\zeta}, 0\right)=i(1-\zeta)\left(M_{1}(\zeta) \bar{S}_{j}+M_{2}(\zeta) \bar{T}_{j}\right)_{k} \quad \text { for } j, k=2, \ldots, n,
\end{aligned}
$$


for some vectors $\left(S_{2}, \ldots, S_{n}\right),\left(T_{2}, \ldots, T_{n}\right) \in \mathbb{C}^{n-1}$. Let $S$ (respectively $T$ ) be the matrix whose columns are $S_{2}, \ldots, S_{n-1}$ (respectively $T_{2}, \ldots, T_{n-1}$ ) and set

$$
N=\left(\begin{array}{cc}
S & \bar{S} \\
T & \bar{T}
\end{array}\right)
$$

We claim that $N$ is invertible. Indeed, since $d G$ is invertible at $(i, O)$, equations (17.4) imply that the only vector $v$ which satisfies the equation $\left(M_{1}(0), M_{2}(0)\right)$. $\left(2 \operatorname{Re}\left(\begin{array}{c}S \\ T\end{array}\right) v\right)=0$ is the zero vector $v=O$. Therefore $S_{2}, \ldots, S_{n}, T_{2}, \ldots, T_{n}$ form a real basis of $\mathbb{C}^{n-1}$.

From this it follows easily that if the vector $\left(\begin{array}{c}v \\ w\end{array}\right)$ belongs to the kernel of $N^{t}$, then $v=w=0$, and thus $N$ is invertible.

Now we are in good shape to compute $\frac{\partial U}{\partial w_{j}}(\xi, O)$. Since $U=u \circ G=\tilde{\lambda}(w)\left(\|w\|^{2}-\right.$ $\operatorname{Im} \xi$ ), from (17.4) we have for $j=1, \ldots, n-1$ and $\operatorname{Im} \xi>0$

$$
-\frac{\partial \tilde{\lambda}}{\partial w_{j}}(O) \operatorname{Im} \xi=\sum_{k=2}^{n}\left[\frac{\partial u}{\partial z_{k}}\left(\frac{\xi-i}{\xi+i}, O\right) \frac{\partial G_{k}}{\partial w_{j}}(\xi, O)+\overline{\frac{\partial u}{\partial z_{k}}\left(\frac{\xi-i}{\xi+i}, O\right) \frac{\partial G_{k}}{\partial \bar{w}_{j}}(\xi, O)}\right] .
$$

Notice that since $u$ is plurisubharmonic in $D$ and harmonic on the complex geodesics whose closure contains $p$, it follows that the functions $\frac{\partial u}{\partial z_{k}}\left(\frac{\xi-i}{\xi+i}, O\right)$ are holomorphic for $\operatorname{Im} \xi>0$. Moreover, by (7.4) both $\frac{\partial G}{\partial w_{j}}(\xi, O)$ and $\frac{\partial G}{\partial \bar{w}_{j}}(\xi, O)$ are holomorphic for $\operatorname{Im} \xi>0$. Taking the real and imaginary part in (7.5) and writing $V\left(=\frac{\partial \tilde{\lambda}}{\partial w}(i, O)\right)=$ $C+i D$ with $C, D \in \mathbb{R}^{n-1}$, we find that there exist two vectors $C^{\prime}, D^{\prime} \in \mathbb{R}^{n-1}$ such that for all $\operatorname{Im} \xi>0$

$$
\begin{aligned}
i C_{j} \xi+i C_{j}^{\prime} & =\sum_{k=2}^{n}\left[\frac{\partial u}{\partial z_{k}}\left(\frac{\xi-i}{\xi+i}, O\right) \frac{\partial G_{k}}{\partial w_{j}}(\xi, O)+\frac{\partial u}{\partial z_{k}}\left(\frac{\xi-i}{\xi+i}, O\right) \frac{\partial G_{k}}{\partial \overline{w_{j}}}(\xi, O)\right], \\
-D_{j} \xi+D_{j}^{\prime} & =\sum_{k=2}^{n}\left[\frac{\partial u}{\partial z_{k}}\left(\frac{\xi-i}{\xi+i}, O\right) \frac{\partial G_{k}}{\partial w_{j}}(\xi, O)-\frac{\partial u}{\partial z_{k}}\left(\frac{\xi-i}{\xi+i}, O\right) \frac{\partial G_{k}}{\partial \overline{w_{j}}}(\xi, O)\right] .
\end{aligned}
$$

Let $V^{\prime}=i C^{\prime}+D^{\prime}$, let $f_{k}(\zeta)=-2 i(1-\zeta)^{2} \frac{\partial u}{\partial z_{k}}(\zeta, O)$ and let $f=\left(f_{1}, \ldots, f_{n}\right)$ for $\zeta \in \mathbb{D}$. Summing (respectively subtracting) (7.6) with (7.7), composing with $\zeta \mapsto i \frac{1+\zeta}{1-\zeta}$, multiplying by $(1-\zeta)$ and using (7.4) we obtain for $\zeta \in \overline{\mathbb{D}} \backslash\{1\}$

$$
\left(\begin{array}{c}
\zeta\left(V+V^{\prime}\right)+\left(V-V^{\prime}\right) \\
\zeta\left(\bar{V}-\bar{V}^{\prime}\right)+\left(\bar{V}+\bar{V}^{\prime}\right)
\end{array}\right)=N^{t}\left(\begin{array}{c}
M_{1}(\zeta)^{t} \\
M_{2}(\zeta)^{t}
\end{array}\right) \cdot f(\zeta)
$$

From this, since $N$ is invertible and also $M_{1}(\zeta), M_{2}(\zeta)$ are invertible for $\zeta$ close to 1 (since by the very definition $M_{1}(1)=\frac{1}{2}$ Id and $M_{2}(1)=\frac{-i}{2} \mathrm{Id}$ ), it follows that $f(\zeta)$ has a limit $L$ at $\zeta=1$ and

$$
\left(\begin{array}{c}
4 V \\
4 \bar{V}
\end{array}\right)=N^{t}\left(\begin{array}{c}
\mathrm{Id} \\
-i \mathrm{ld}
\end{array}\right) L .
$$

Therefore $\left(S^{t}-i T^{t}\right) L-\left(S^{t}+i T^{t}\right) \bar{L}=O$. Writing $L=\alpha+i \beta$ for $\alpha, \beta \in \mathbb{R}^{n-1}$, we have $S^{t} \beta-T^{t} \alpha=O$, and, since $\alpha, \beta$ are real, this is equivalent to

$$
N^{t}\left(\begin{array}{c}
\beta \\
-\alpha
\end{array}\right)=O \text {. }
$$

But $N$ is invertible and therefore $\alpha=\beta=O$, which means $L=O$. Finally, from (7.8) it follows that $V=O$. 
The pluricomplex Poisson kernel can also be characterized in terms of its level sets:

Proposition 7.4. Let $D \subset \mathbb{C}^{n}$ be a bounded strongly convex domain with smooth boundary and let $p \in \partial D$. Let $u \in \operatorname{Psh}(D) \cap L_{l o c}^{\infty}(D)$ be such $(\partial \bar{\partial} u)^{n}=0$ in $D$ and $\lim _{z \rightarrow x} u(z)=0$ for all $x \in \partial D \backslash\{p\}$. If $u$ has the same level sets as $\Omega_{D, p}$, then there exists $c>0$ such that $u=c \Omega_{D, p}$.

Proof. By hypothesis there exists a function $Y: \mathbb{R}^{-} \rightarrow \mathbb{R}^{-}$such that $u(z)=$ $Y\left(\Omega_{D, p}(z)\right)$ for all $z \in D$. We need to show that there exists $c>0$ such that $Y(t)=c t$ for all $t \in \mathbb{R}^{-}$. To this aim, since each complex geodesic whose closure contains $p$ intersects every horosphere, it is enough to prove that $u(z)=c \Omega_{D, p}(z)$ for $z$ belonging to any complex geodesic whose closure contains $p$.

Let $S$ be a complex geodesic in $D$ such that $p \in \bar{S}$ and $\rho: D \rightarrow S$, the associated Lempert's projection. We can assume that $D$ is linearized along $S$ in Lempert's coordinates. Let $\tilde{B}$ be an open disc relatively compact in $S$. Let

$$
\mathcal{P}:=\left\{\begin{array}{l}
\tilde{v} \in \operatorname{subh}(\tilde{B}), \\
\lim _{\sup _{\zeta \rightarrow x}} \tilde{v}(\zeta) \leq u(x) \quad \forall x \in \partial \tilde{B} .
\end{array}\right.
$$

If we prove that $\left.u\right|_{\tilde{B}}$ is the maximum of $\mathcal{P}$, then by the arbitrariness of $\tilde{B}$ it follows that $u$ is harmonic on $S$. Therefore $u \circ \varphi$ is harmonic and negative in $\mathbb{D}$ and it is zero on $\partial \mathbb{D} \backslash\{1\}$; hence it is a constant multiple of the Poisson kernel of $\mathbb{D}$. That is, there exists $c>0$ such that $u(\varphi(\zeta))=c \Omega_{D, p}(\varphi(\zeta))$ for all $\zeta \in \mathbb{D}$, as wanted.

In order to prove that $\left.u\right|_{\tilde{B}}$ is the maximum of $\mathcal{P}$, let $\epsilon>0$ small. Let $T=$ $\rho^{-1}(\tilde{B}) \cap D$ and let $B=\{z \in T: \operatorname{dist}(z, \partial D)>\epsilon\}$ (a cylinder in $D$ ). The boundary of the set $B$ is made of two parts: $R_{1}$ which has the property that $\rho\left(R_{1}\right)=\partial \tilde{B}$, and $R_{2}$ (the bottom and top of the cylinder) such that $\rho\left(R_{2}\right) \subset \tilde{B} ; \partial B=R_{1} \cup R_{2}$. Since $u=0$ on $\partial D$ and $p \notin T$, we can choose $\epsilon$ so small that $\inf _{x \in R_{2}} u(x)>\max _{x \in \partial \tilde{B}} u(x)$.

Let $\tilde{v} \in \mathcal{P}$, and let $v:=\left.\tilde{v} \circ \rho\right|_{B}$. Then $v$ is plurisubharmonic in $B$ and $\sup _{x \in B} v(x)$ $=\sup _{x \in \partial \tilde{B}}\left(\lim \sup _{z \rightarrow x} v(z)\right)$. In particular by construction $\lim _{\sup _{z \rightarrow x}} v(z) \leq u(x)$ for all $x \in R_{2}$. Also, we have that $\lim \sup _{B \ni z \rightarrow x} v(z)=\limsup _{B \ni z \rightarrow x} \tilde{v}(\rho(z)) \leq$ $u(\rho(x))$ for all $x \in R_{1}$. Now $u$ has the same level sets as $\Omega_{D, p}$, and thus by (3.3) we have that $u(x) \geq u(\rho(x))$ for all $x \in D$ and hence $\lim _{\sup _{B \ni z \rightarrow x} v(z) \leq u(x) \text { for all }}$ $x \in R_{1}$. Therefore $\limsup _{B \ni z \rightarrow x} v(z) \leq u(x)$ for all $x \in \partial B$, and by the maximality of Monge-Ampere solutions, it follows that $v \leq u$ in $B$ and in particular $\tilde{v} \leq\left. u\right|_{\tilde{B}}$, and the arbitrariness of $\tilde{v}$ implies that $\left.u\right|_{\tilde{B}}$ is maximal in $\mathcal{P}$.

The previous argument, together with Theorem 7.3, shows that if $u \in \operatorname{Psh}(D) \cap$ $C^{2}(D)$ is such that $(\partial \bar{\partial} u)^{n}=0$ on $D$ and $\lim _{z \rightarrow x} u(z)=0$ for all $x \in \partial D \backslash\{p\}$, then $u=c \Omega_{D, p}$ for some $c>0$ if and only if $u(\rho(z)) \leq u(z)$ for all $z \in D$ and for all Lempert's projections $\rho$.

More generally, if $f: D \rightarrow D$ is holomorphic and $f(p)=p$ as a non-tangential limit we can define the boundary dilatation coefficient $\alpha_{f}(p)$ of $f$ at $p$ by means of

$$
\frac{1}{2} \log \alpha_{f}(p):=\liminf _{z \rightarrow p}\left[k_{D}\left(z_{0}, z\right)-k_{D}\left(z_{0}, f(z)\right)\right] .
$$

It turns out that $\alpha_{f}(p)>0$ and, if $\alpha_{f}(p)<\infty$, we can rephrase Abate's generalization of the classical Julia Lemma (see [1], 2]) saying that $\alpha_{f}(p) f^{*}\left(\Omega_{D, p}\right) \leq \Omega_{D, p}$. In [11, Theorem 7.3], with the assumption of slightly more regularity of $f$ at $p$, it is proved that $f$ is an automorphism of $D$ if and only if $f^{*}\left(\Omega_{D, p}\right)=\alpha_{f}(p) \Omega_{D, p}$. 
Using Abate's version of the Julia-Wolff-Caratheodory theorem for strongly convex domains (see [1, [4]), it is easy to see that $\alpha_{\rho}(p)=1$ for all Lempert's projections $\rho$. Therefore, the above discussion shows that the property $f^{*}\left(\Omega_{D, p}\right) \leq$ $\alpha_{f}(p) \Omega_{D, p}$ characterizes $\Omega_{D, p}$. In other words:

Proposition 7.5. Let $D \subset \mathbb{C}^{n}$ be a bounded strongly convex domain with smooth boundary and let $p \in \partial D$. Let $u \in \operatorname{Psh}(D) \cap C^{2}(D)$ be such that $(\partial \bar{\partial} u)^{n}=0$ in $D$ and $\lim _{z \rightarrow x} u(z)=0$ for all $x \in \partial D \backslash\{p\}$. Then there exists $c \geq 0$ such that $u=c \Omega_{D, p}$ if and only if for all $f: D \rightarrow D$ holomorphic such that $f(p)=p$ as a non-tangential limit and $\alpha_{f}(p)<\infty$, it follows that

$$
\alpha_{f}(p) f^{*}(u) \leq u \text {. }
$$

Some remarks about uniqueness properties are in order. First, it would be interesting to see whether Theorem 7.3 (and thus its corollaries) holds without any regularity hypothesis on $u$. A direct argument using the sub-media property of plurisubharmonic functions shows that Theorem 7.3 holds in the unit ball $\mathbb{B}^{n}$ with no regularity hypothesis on $u$. Such an argument seems however to fail in general.

Another (maybe more) interesting open question is the following:

Question 7.6. Let $D \subset \mathbb{C}^{n}$ be a bounded strongly convex domain with smooth boundary and let $p \in \partial D$. Let $u \in \operatorname{Psh}(D) \cap L_{l o c}^{\infty}(D)$ be such that $(\partial \bar{\partial} u)^{n}=0$ in $D$ and $\lim _{z \rightarrow x} u(z)=0$ for all $x \in \partial D \backslash\{p\}$. Is it true that $u=c \Omega_{D, p}$ for some constant $c \geq 0$ ?

As we already recalled, the answer to such a question is "yes" in the case where $D=\mathbb{D}$ is the unit disc, $u<0$ in $\mathbb{D}$ and $\Omega_{D, p}$ is the (negative) Poisson kernel.

\section{REPRODUCING FORMULAS}

Let $D$ be a bounded strongly convex domain in $\mathbb{C}^{n}$ with smooth boundary. As usual, let $d^{c}:=i(\bar{\partial}-\partial)$. Let $r$ be a defining function of $D$ and let $\omega_{D}$ be the real $(2 n-1)$-form defined as

$$
\omega_{\partial D}:=\left.\frac{\left(d d^{c} r\right)^{n-1} \wedge d^{c} r}{\|d r\|^{n}}\right|_{\partial D} .
$$

Such a form $\omega_{\partial D}$ is positive, and it is easily seen to be independent of the defining function $r$ chosen to define it.

Let $L_{D, z_{0}}$ denote the Lempert solution of (0.1) and denote by $\Omega_{D, p}$ the solution of (0.2) with singularity at $p \in \partial D$ given by Theorem 0.1 . From the very definition of $\Omega_{D, p}$ and since the boundary spherical representation $\Phi_{p}$ of Chang-Hu-Lee is smooth out of the diagonal of $\partial D \times \partial D$ as the vertex $p$ varies on $\partial D$ (see [13, Theorem 3]), it follows that the map $\bar{D} \times \partial D \ni(z, p) \mapsto \Omega_{D, p}(z) \in \mathbb{R}$ is $C^{\infty}$ on $(\bar{D} \times \partial D) \backslash\{(p, p) \in \partial D \times \partial D\}$.

We briefly recall Demailly's theory [14, 15]. Let $\varphi \in \operatorname{Psh}(D)$ be such that $\exp (\varphi) \in C^{0}(\bar{D})$, that $\varphi<0$ on $D$ and that $\varphi=0$ on $\partial D$. Let $R<0$ and let $B_{R}=\{z \in D: \varphi(z)<R\}$. Moreover let $S_{R}=\partial B_{R}$ and $\varphi_{R}(z)=\max \{\varphi(z), R\}$. By [15, (1.4)] we can write

$$
\left(d d^{c} \varphi_{R}\right)^{n}=\mathbf{1}_{\mathbb{C}^{n} \backslash B_{R}}\left(d d^{c} \varphi\right)^{n}+\mu_{\varphi, R}
$$

where $\mathbf{1}_{\mathbb{C}^{n} \backslash B_{R}}$ is the characteristic function of $\mathbb{C}^{n} \backslash B_{R}$ and $\mu_{\varphi, R}$ is a positive measure supported on $S_{R}$. By [15, Théorème 3.1] if the total Monge-Ampère mass of $\varphi$ is 
finite, i.e., if $\int_{D}\left(d d^{c} \varphi\right)^{n}<+\infty$, then as $R \rightarrow 0$ the measures $\mu_{\varphi, R}$ converge weakly on $\mathbb{C}^{n}$ to a positive measure $\mu_{\varphi}$ supported on $\partial D$, with total mass $\int_{D}\left(d d^{c} \varphi\right)^{n}$. We denote by $\mu_{z}$ the limit measure of $L_{D, z}$. By [15, Théorème 5.1] it follows that for all $F \in \operatorname{Psh}(D) \cap C^{0}(\bar{D})$ we have the following representation formula:

$$
F(z)=\mu_{z}(F)-\frac{1}{2 \pi^{n}} \int_{w \in D}\left|L_{D, z}(w)\right| d d^{c} F(w) \wedge\left(d d^{c} L_{D, z}\right)^{n-1}(w) .
$$

We can prove the following result:

Theorem 8.1. Let $D$ be a bounded strongly convex domain in $\mathbb{C}^{n}$ with smooth boundary. Then

$$
d \mu_{z}(p)=\left|\Omega_{D, p}(z)\right|^{n} \omega_{\partial D}(p) .
$$

Proof. First of all, since $L_{D, z}$ is $C^{\infty}$ on $\bar{D} \backslash\{z\}$ and $\left.d L_{D, z}\right|_{\partial D} \neq 0$, arguing as in [15] we see that

$$
d \mu_{z}=\left.\left(d d^{c} L_{D, z}\right)^{n-1} \wedge d^{c} L_{D, z}\right|_{\partial D}
$$

From (6.2) we have

$$
\left|\Omega_{D, p}(z)\right|=\left\|\frac{\partial L_{D, z}}{\partial \nu_{p}}(p)\right\|=\left\|d\left(L_{D, z}\right)_{p}\right\|,
$$

where the last equality follows from $\left.L_{D, z}\right|_{\partial D}=0$ which implies that $d\left(L_{D, z}\right)_{p}$ is a positive multiple of $\nu_{p}$, the unit normal to $\partial D$ at $p \in \partial D$ (here, as usual and with an abuse of notation, we identify the gradient of a function with its differential). Thus

$$
d \mu_{z}=\left.\left|\Omega_{D, p}(z)\right|^{n} \frac{\left(d d^{c} L_{D, z}\right)^{n-1} \wedge d^{c} L_{D, z}}{\left\|d L_{D, z}\right\|^{n}}\right|_{\partial D} .
$$

To end the proof we only need to check that

$$
\omega_{\partial D}=\left.\frac{\left(d d^{c} L_{D, z}\right)^{n-1} \wedge d^{c} L_{D, z}}{\left\|d L_{D, z}\right\|^{n}}\right|_{\partial D} .
$$

To this aim, it is enough to show that if $r$ is a (local) defining function for $D$ on a neighborhood $U_{p}$ of $p \in \partial D$, then $L_{D, z}=h \cdot r$ on $U_{p} \cap \bar{D}$ for some positive $h \in C^{\infty}\left(U_{p} \cap \bar{D}\right)$. Then a direct computation gives the result. Up to changes of coordinates we can assume that $U_{p} \cap D=\left\{(x, y) \in \mathbb{C} \times \mathbb{C}^{n-1}: x<0\right\}$. Thus $L_{D, z}(x, y) / x$ is defined and positive on $U_{p} \cap D$. If we let $h(x, y)=\int_{0}^{1} \frac{\partial L_{D, z}}{\partial x}(t x, y) d t$, then $h$ is $C^{\infty}\left(U_{p} \cap \bar{D}\right)$ and coincides with $L_{D, z}(x, y) / x$ in $U_{p} \cap D$. Moreover, since $d L_{D, z} \neq 0$ on $\partial D$ it follows that $h>0$ on $U_{p} \cap \bar{D}$.

From (8.1) and Theorem 8.1 we obtain:

Theorem 8.2. Let $F \in \operatorname{Psh}(D) \cap C^{0}(\bar{D})$. Then for all $z \in D$

$$
\begin{aligned}
F(z) & =\int_{p \in \partial D}\left|\Omega_{D, p}(z)\right|^{n} F(p) \omega_{\partial D}(p) \\
& -\frac{1}{2 \pi^{n}} \int_{w \in D}\left|L_{D, z}(w)\right| d d^{c} F(w) \wedge\left(d d^{c} L_{D, z}\right)^{n-1}(w) .
\end{aligned}
$$

In particular if $F$ is pluriharmonic, then

$$
F(z)=\int_{p \in \partial D}\left|\Omega_{D, p}(z)\right|^{n} F(p) \omega_{\partial D}(p) .
$$


Remark 8.3. If $F \in C^{2}(\bar{D})$ (but not plurisubharmonic in $D$ ), then there exists $C>0$ such that $F(z)+C\|z\|^{2} \in \operatorname{Psh}(D) \cap C^{0}(\bar{D})$. Thus Theorem 8.2 applies and one gets

$$
\begin{aligned}
F(z)+ & C\|z\|^{2}=\int_{p \in \partial D}\left|\Omega_{D, p}(z)\right|^{n} F(p) \omega_{\partial D}(p) \\
& +C \int_{p \in \partial D}\left|\Omega_{D, p}(z)\right|^{n}\|p\|^{2} \omega_{\partial D}(p) \\
& -\frac{1}{2 \pi^{n}} \int_{w \in D}\left|L_{D, z}(w)\right| d d^{c} F(w) \wedge\left(d d^{c} L_{D, z}\right)^{n-1}(w) \\
& -C \frac{1}{2 \pi^{n}} \int_{w \in D}\left|L_{D, z}(w)\right| d d^{c}\|w\|^{2} \wedge\left(d d^{c} L_{D, z}\right)^{n-1}(w) \\
& =\int_{p \in \partial D}\left|\Omega_{D, p}(z)\right|^{n} F(p) \omega_{\partial D}(p) \\
& -\frac{1}{2 \pi^{n}} \int_{w \in D}\left|L_{D, z}(w)\right| d d^{c} F(w) \wedge\left(d d^{c} L_{D, z}\right)^{n-1}(w)+C\|z\|^{2} .
\end{aligned}
$$

Therefore Theorem 8.2 applies to any $F \in C^{2}(\bar{D})$ (not necessarily plurisubharmonic). As a consequence, it follows that the kernel given by $\left|\Omega_{D, p}(z)\right|^{n} \omega_{\partial D}(p)$ is the unique reproducing kernel associated to $L_{D, z}$; namely, (8.1) cannot hold with any other measure $T_{z}$ in place of $\mu_{z}$.

\section{REFERENCES}

[1] M. Abate, Iteration theory of holomorphic maps on taut manifolds. Mediterranean Press, Rende, Cosenza, 1989. MR1098711 (92i:32032)

[2] M. Abate, Horospheres and iterates of holomorphic maps. Math. Z. 198 (1988), 225-238. MR.939538 (90e:32028)

[3] M. Abate, Common fixed points of commuting holomorphic maps. Math. Ann. 283 (1989), 645-655. MR990593 (90k:32074)

[4] M. Abate, The Lindelöf principle and the angular derivative in strongly convex domains. J. Analyse Math. 54 (1990), 189-228. MR.1041181 (91d:32011)

[5] R. Abraham, J. E. Marsden, T. Ratiu, Manifolds, tensor analysis, and applications, Second edition, Springer, New York, 1988. MR960687 (89f:58001)

[6] E. Bedford, Survey of pluri-potential theory. Several Complex Variables. Proceedings of the Mittag-Leffler Institute 1987-1988. Math. Notes 38, Princeton Univ. Press, Princeton, 1993, 48-97. MR:1207855 (94b:32014)

[7] E. Bedford, B.A. Taylor, The Dirichlet problem for the complex Monge-Ampère equation. Invent. Math. 37 (1976), 1-44. MR0445006 (56:3351)

[8] E. Bedford, B.A. Taylor, A new capacity for plurisubharmonic funtions. Acta Math. 149 (1982), 1-41. MR674165 (84d:32024)

[9] J. Bland, T. Duchamp, M. Kalka, On the automorphism group of strictly convex domains in $\mathbb{C}^{n}$. Contemp. Math. 49 (1986), 19-30. MR833801 (87f:32060)

[10] F. Bracci, Dilatation and order of contact for holomorphic self-maps of strongly convex domains, Proc. London Math. Soc., 86, 1 (2003), 131-152. MR1971466 (2004b:32022)

[11] F. Bracci, G. Patrizio, Monge-Ampère foliations with singularities at the boundary of strongly convex domains, Math. Ann., 332, 3 (2005) 499-522. MR2181760 (2006j:32048)

[12] H. Busemann, The Geometry of Geodesics, Academic Press, New York, 1955. MR0075623 $(17: 779 \mathrm{a})$

[13] C.H. Chang, M.C. Hu, H.P. Lee, Extremal analytic discs with prescribed boundary data. Trans. Amer. Math. Soc. 310, 1 (1988) 355-369. MR930081 (89f:32043)

[14] J.-P. Demailly, Mesures de Monge-Ampère et caractérisation géométrique des variétés algébriques affines Mém. Soc. Math. France (N.S.) No. 19 (1985). MR813252 (87g:32030) 
[15] J.-P. Demailly, Mesures de Monge-Ampère et mesures pluriharmoniques. Math. Z. 194 (1987), 519-564. MR881709 (88g:32034)

[16] J. Globevnik, Perturbation by analytic discs along maximal real submanifolds of $\mathbb{C}^{N}$. Math. Z. 217, 2 (1994), 287-316. MR1296398 (95j:32031)

[17] L. Hörmander, Notions of convexity. Birkhäuser, Boston, Basel, Berlin, 1994. MR1301332 (95k:00002)

[18] X. Huang, A non-degeneracy property of extremal mappings and iterates of holomorphic self-mappings. Ann. Scuola Norm. Sup. Pisa Cl. Sci. (4) 21, 3 (1994), 399-419. MR1310634 (95k:32025)

[19] X. Huang, A preservation principle of extremal mappings near a strongly pseudoconvex point and its applications. Illinois J. Math. 38, 2 (1994), 283-302. MR1260844 (95a:32043)

[20] M. Klimek, Pluripotential theory. London Math. Soc. Monographs, Oxford University Press, 1991. MR:1150978 (93h:32021)

[21] L. Lempert, La métrique de Kobayashi et la representation des domaines sur la boule. Bull. Soc. Math. Fr. 109 (1981), 427-474. MR660145 (84d:32036)

[22] L. Lempert, Holomorphic retracts and intrinsic metrics in convex domains. Analysis Math. 8 (1982), 257-261. MR690838 (84f:32026)

[23] L. Lempert, Intrinsic distances and holomorphic retracts. Complex Analysis and Applications '81, Sofia (1984), 341-364. MR883254 (87m:32051)

[24] L. Lempert, Solving the degenerate Monge-Ampère equation with one concentrated singularity. Math. Ann. 263 (1983), 515-532. MR707246 (84k:32024)

[25] K.-W. Leung, G. Patrizio, P.-M. Wong, Isometries of intrinsic metrics on strictly convex domains. Math. Z. 196 (1987), 343-353. MR913661 (89c:32047)

[26] M. Y. Pang, Smoothness of the Kobayashi metric of non-convex domains, Internat. J. Math. 4, 6 (1993) 953-987. MR1250257 (95d:32027)

[27] M. Y. Pang, Pseudoconvex domains in $\mathbb{C}^{2}$ with convexifiable boundary around an extremal disk, Math. Z. 226, 4 (1997), 513-532. MR1484708 (98m:32023)

[28] G. Patrizio, Parabolic exhaustion for strictly convex domains. Manuscr. Math. 47 (1984), 271-309. MR744324 (85k:32044)

[29] G. Patrizio, A characterization of complex manifolds biholomorphic to a circular domain. Math. Z. 189 (1985), 343-363. MR783561 (86j:32002)

[30] T. Ransford, Potential theory in the complex plane. London Mathematical Society Student Texts, 28. Cambridge University Press, Cambridge, 1995. MR1334766|(96e:31001)

[31] J. Siciak, On some extremal functions and their applications in the theory of analytic functions of several complex variables. Trans. Amer. Math. Soc. 105 (1962), 322-357. MR0143946 $(26: 1495)$

[32] A. Spiro, S. Trapani, Eversive maps of bounded convex domains in $\mathbb{C}^{n+1}$. The Journal of Geom. Anal. 12, 4 (2002), 695-715. MR1916865(2003e:32022)

[33] S. Trapani, Defect and evaluations. J. Geom. Anal. 10, 4 (2000), 739-758. MR.1817784 (2002b:32018)

[34] S. Trapani, Dual maps and Kobayashi distance of bounded convex domains in $\mathbb{C}^{n}$. Proc. of the Workshop "Contemporary Geometry and Related Topics", Belgrade, Jugoslavia 2002. World Scientific, 2004. MR2070882 (2005f:32023)

[35] J.-M. Trépreau, On the global Bishop equation, manuscript.

[36] S. M. Webster, On the reflection principle in several complex variables. Proc. Amer. Math. Soc. 71, 1 (1978), 26-28. MR0477138(57:16681)

Dipartimento di Matematica, Università di Roma "Tor Vergata", Via della Ricerca Scientifica 1, 00133 Roma, Italy.

E-mail address: fbracci@mat.uniroma2.it

Dipartimento di Matematica "Ulisse Dini", Università di Firenze, Viale Morgagni 67-A, 50134 Firenze, Italy.

E-mail address: patrizio@math.unifi.it

Dipartimento di Matematica, Università di Roma "Tor Vergata”, Via della Ricerca Scientifica 1, 00133 Roma, Italy.

E-mail address: trapani@mat.uniroma2.it 